\title{
PRICES, PRODUCT DIFFERENTIATION, AND HETEROGENEOUS SEARCH COSTS
}

\author{
José L. Moraga-González \\ Zsolt Sándor \\ Matthijs R. Wildenbeest
}


The Public-Private Sector Research Center is a Research Center based at IESE Business School. Its mission is to develop research that analyses the relationships between the private and public sectors primarily in the following areas: regulation and competition, innovation, regional economy and industrial politics and health economics.

Research results are disseminated through publications, conferences and colloquia. These activities are aimed to foster cooperation between the private sector and public administrations, as well as the exchange of ideas and initiatives.

The sponsors of the Public-Private Sector Research Center are the following:

- Ajuntament de Barcelona

- Departament d' Economia i Coneixement de la Generalitat de Catalunya

- Departament d' Empresa i Ocupació de la Generalitat de Catalunya

- Diputació de Barcelona

- Fundació AGBAR

- Institut Català de les Empreses Culturals (ICEC)

- PricewaterhouseCoopers

- Sanofi

- FGC

The contents of this publication reflect the conclusions and findings of the individual authors, and not the opinions of the Center's sponsors. 


\title{
Prices and Heterogeneous Search Costs*
}

\author{
José L. Moraga-González ${ }^{\dagger}$ \\ Zsolt Sándor \\ Matthijs R. Wildenbeest ${ }^{\S}$ \\ First draft: July 2014 \\ Revised: June 2015
}

\begin{abstract}
We study price formation in a model of consumer search for differentiated products when consumers have heterogeneous marginal search costs. We provide conditions under which a symmetric Nash equilibrium exists and is unique. Search costs affect two margins - the intensive search margin (or search intensity) and the extensive search margin (or the decision to search rather than to not search at all). These two margins affect the elasticity of demand in opposite directions and whether lower search costs result in higher or lower prices depends on the properties of the search cost density. When the search cost density has the increasing likelihood ratio property (ILRP), the effect of lowering search costs on the intensive search margin has a dominating influence and prices decrease. By contrast, when the search cost density has the decreasing likelihood ratio property (DLRP), the effect on the extensive search margin is dominant and lower search costs result in higher prices. We compare these results with those obtained when consumers have heterogeneous fixed search costs.
\end{abstract}

Keywords: sequential search, search cost heterogeneity, differentiated products, monotone likelihood ratio

JEL Classification: D43, D83, L13

\footnotetext{
*Earlier versions of this paper were circulated under the title "Do higher search costs make markets less competitive?" We would like to thank Simon Anderson, Guillermo Caruana, Alex de Cornière, Knut-Eric Joslin, Silvana Krasteva, Theo Dijkstra, Dmitry Lubensky, Andras Niedermayer, Marielle Non, Vaiva Petrikaite, Régis Renault, Mariano Tappata, Makoto Watanabe, and Jidong Zhou for their useful remarks. Special thanks go to Paulo K. Monteiro for his comments, help and useful references. The paper has also benefited from presentations at University Carlos III Madrid, University of Copenhagen, CEMFI (Madrid), the Netherlands Bureau for Economic Policy Analysis (CPB), Paris School of Economics, Sauder Business School at UBC, University of East Anglia, University of Mannheim, University of Oxford, the Fifth Workshop on Search and Switching Costs (Bloomington, IN), the IIOC 2014 Conference (Chicago), the 2014 MaCCI Workshop on Consumer Search in Bad Homburg, and the SaM 2015 Workshop at the University of Bristol. Financial support from the Marie Curie Excellence Grant MEXT-CT-2006-042471, grant ECO2011-29533 of the Spanish Ministry of Economy and Competitiveness, and grant PN-II-ID-PCE-2012-4-0066 of the Romanian Ministry of National Education, CNCS-UEFISCDI, is gratefully acknowledged.

${ }^{\dagger}$ Address for correspondence: VU University Amsterdam, Department of Economics, De Boelelaan 1105, 1081HV Amsterdam, The Netherlands. E-mail: j.1.moragagonzalez@vu.nl. Moraga is also affiliated with the University of Groningen, the Tinbergen Institute, the CEPR, and the Public-Private Sector Research Center (IESE, Barcelona).

${ }^{\ddagger}$ Sapientia University Miercurea Ciuc, Romania, and Affiliate Fellow at CERGE-EI, Prague. E-mail: zsosan@gmail.com.

${ }^{\S}$ Kelley School of Business, Indiana University, USA. E-mail: mwildenb@indiana.edu.
} 


\section{Introduction}

Throughout history, technological improvements have made it possible for consumers to participate in markets that were previously beyond their reach. A recent example of such a technological development is the Internet. The introduction and sustained growth of e-commerce has significantly lowered the transaction costs consumers experience while shopping. This has not only made it easier for consumers to search for and compare products but it has also made it easier for consumers to get access to new markets and hard-to-find products. This has also led to the Internet's Long Tail phenomenon: the Internet allows consumers to access a much larger product selection, including niche products that were previously difficult to find, thereby creating a long tail in the sales distribution (see, e.g., Brynjolfsson, $\mathrm{Hu}$, and Simester, 2011). ${ }^{1}$

How are technological advances that make it easier for consumers to search expected to affect market competitiveness? The conventional answer is that a reduction in search costs increases the elasticity of demand and as such reduces prices. However, as illustrated by the example, this is not the complete story. Lower search costs also allow consumers to search for products that previously were not part of their consideration sets. Since these new consumers are likely to have higher search costs (because otherwise they would have been actively searching before), this leads to compositional changes in the consumer population that may potentially decrease the elasticity of demand. More consumer participation as a result of a technology-driven reduction in search costs may end up being the dominating factor, which means firms will raise prices, even when search costs go down for all consumers.

Hortaçsu and Syverson (2004) present empirical evidence that is consistent with this particular mechanism in their study of the US mutual fund industry during the late nineties. Not that long ago investors had to go through significant effort to search for adequate investment opportunities while managing their financial investments. However, the rise of Internet banking and online brokerages during the late nineties decreased transaction costs and made it easier for individuals to search for new investment opportunities. Hortaçsu and Syverson (2004) find that during this period investment fund fees went up and that search costs decreased at the lower percentiles of the search cost distribution while increased at the upper percentiles. According to the authors, this surprising finding (i.e., a second-order stochastic dominance decrease in search costs leading to a price rise) is explained by the increase in the number of households

\footnotetext{
${ }^{1}$ The expansion of the railroad network in the United States in the 19th century and the liberalization of airlines in the 20th century are events that also led to significantly lower transportation costs and greater market integration. As with the Internet, lower transportation costs were not only beneficial to consumers because of reduced transaction costs but also because they allowed consumers to be active in new markets.
} 
that participated in the mutual fund market for the first time. These new investors arguably had higher search costs and were less savvy. Hortaçsu and Syverson argue that the entry of these new investors changed the composition of the investor population and made demand more inelastic, explaining the increase in mutual fund fees.

The main objective of our paper is to understand under which conditions a mechanism like the one described above occurs. In particular, our goal is to derive general conditions under which a first- or second-order stochastic dominance decrease in search costs triggers sufficient consumer entry such that the elasticity of demand is lowered and equilibrium prices go up. In order to do so, we develop a model of consumer search for differentiated products in which consumers have heterogeneous search costs and the decision to participate in the market is endogenous. Our model, which is presented in Section 2, builds on the seminal model of consumer search for differentiated products introduced by Wolinsky (1986), and further studied by Anderson and Renault (1999). ${ }^{2}$ We extend this model by allowing for arbitrary search cost densities. This is crucial for studying the second-order stochastic dominance (SOSD) decreases in search costs observed in the mutual fund industry by Hortaçsu and Syverson (2004).

Section 3 deals with marginal search cost heterogeneity and characterizes the symmetric Nash equilibrium of the model. Our first contribution is the study of the existence and uniqueness of a symmetric Nash equilibrium in pure strategies. Because a direct verification of the second order conditions fails to deliver clear-cut results we proceed by studying the conditions under which the profit function of a typical firm is quasi-concave. We show that when the density of search costs is sufficiently log-concave, an equilibrium exists and is unique. In order to prove our existence result we use an aggregation theorem due to Prékopa (1973) that shows that integration preserves log-concavity (see also Caplin and Nalebuff, 1991, p.46).

Our second contribution is the study of the comparative statics effects of lower search costs. In Section 4 we show that a change in search costs affects two margins, namely, the intensity with which consumers search (which we call the intensive search margin) and the share of consumers who choose to search for a good deal in the first place (which we refer to as the extensive search margin). By assuming that all consumers search at least once, the literature has typically focused on the effects of the intensive search margin on price determination and

\footnotetext{
${ }^{2}$ Arguably Wolinsky's framework has become the workhorse model of consumer search for differentiated products. Recent work that builds on this article include Bar-Isaac, Caruana, and Cunat (2012), who extend the model to the case of quality-differentiated firms and study design differentiation, Armstrong, Vickers, and Zhou (2009), who study search and pricing behavior in the presence of a prominent firm, Haan and Moraga-González (2011a), who study the emergence and the price effects of prominence, Moraga-González and Petrikaite (2013), who examine the effect of search costs on mergers, and Zhou (2014), who studies multi-product search.
} 
has thereby neglected the role of the extensive search margin. ${ }^{3}$

Recognizing that prices do not only affect the intensive search margin but may also affect the extensive search margin turns out to be critical for a complete understanding of the functioning of consumer search markets. We show that if the extensive margin does not play a role, any decrease in search costs in the sense of first-order stochastic dominance (FOSD) results in a lower equilibrium price. This would happen when the range of search costs is sufficiently small and all consumers choose to search in market equilibrium. Consumers, facing lower search costs, all choose to search more and demand products that are more attractive. Firms, anticipating that average demand becomes more elastic, respond by reducing their prices. Under certain conditions on the density of match values (satisfied by for example the uniform distribution), a decrease in search costs in the sense of second-order stochastic dominance (SOSD) also results in lower prices.

If the extensive search margin does play a role, we get very different results. Specifically, we show that a shock that reduces search costs leads to more search but also brings new consumers into the market with relatively high search costs. We establish that for search cost densities with the decreasing likelihood ratio property (DLRP), a decrease in search costs in the sense of FOSD or SOSD results in a higher equilibrium price. That is, for densities with the DLRP a decrease in search costs has a bigger effect on the extensive search margin than on the intensive search margin, and as a result prices go up. This is exactly what seems to have occurred in Hortaçsu and Syverson's (2004) study of the mutual fund industry: easier search led to entry of new consumers and this resulted in a more inelastic composition of demand so prices went up in spite of the increasing popularity of Internet banking and online trading. For search cost densities with the increasing likelihood ratio property (ILRP) we find the opposite: a decrease in search costs leads to lower prices. For this type of densities the effect of a fall in search costs on search intensity dominates the effect on demand composition.

We believe that this mechanism may also be important for understanding other existing empirical evidence regarding the effects of the Internet on retail price competition. The commonly held belief is that the Internet improves consumer search technology and this should naturally lead to lower prices online than offline. Despite this belief, the empirical evidence is mixed: although some studies indeed find lower prices online than offline (Brynjolfsson and Smith, 2000; Scott Morton, Zettelmeyer, Silva-Risso, 2001), others find no effects or even the opposite (Clay,

\footnotetext{
${ }^{3}$ This point is also the central tenet in Anderson and Renault (2006), who, by allowing for arbitrary search costs, are able to reconcile the empirical observation that much of the advertising we observe in arguably search environments does impart only match information and not price information.
} 
Krishnan, and Wolff, 2001; Clemons, Hann, and Hitt, 2002; Goolsbee, 2001; Lee, 1998). The entry of new buyers as a result of a reduction in transaction costs due to the Internet may very well be part of the explanation for why some studies have found online prices to be higher than offline prices.

A third contribution of our paper is more technical in nature and relates to the properties of truncated densities. Specifically, as part of our proofs for our findings on the comparative statics effects of lower search costs we show that for any two densities that can be ranked according to the ILRP, the corresponding distributions truncated from above can be ranked according to the FOSD criterion, irrespective of the location of the truncation point. Conversely, if the densities can be ranked according to the DLRP, then the corresponding distributions truncated from above can be ranked according to the reverse FOSD. To prove this result we use a tool from the statistics literature by Ahlswede and Daykin (1978) that has also been used by for instance Athey (2002), although for a different application.

Finally, in Section 5 we study what happens to prices when there is a change in the distribution of fixed search costs rather than marginal search costs. We show that when consumers differ in their fixed costs of search (but face a similar marginal cost of search) we obtain quite different results because a decrease in the fixed costs of search results in an increase in demand without altering the elasticity of demand. As a result, prices do not change. This illustrates an important point: changes in the size of the market do not always lead to changes in demand elasticity. The mechanism by which prices are affected by changes in the extensive search margin goes via the changes in the gains from search of the average consumer who chooses to search. Changes in the market that do not affect the gains from search of the average consumer (as happens when fixed search costs vary while marginal search costs are fixed) do not have a bearing on the elasticity of demand, which implies that prices do not change.

\section{Related literature}

Most of the previous literature has proceeded under the restrictive assumption that search costs are required to be "low enough," de facto implying that all consumers choose to search at least once in equilibrium rather than to not search at all (e.g., Stahl, 1989; Burdett and Judd, 1983; Wolinsky, 1986). As pointed out by Stiglitz (1979), the alternative assumption that search costs are large may cause the market to collapse. ${ }^{4}$ As we have discussed before, this unravelling of

\footnotetext{
${ }^{4}$ For example, in the setting of Diamond (1971), the only price that can be part of a market equilibrium is the monopoly price (the well-known "Diamond paradox"). If the search cost is relatively high, the surplus consumers derive at the monopoly price may be insufficient to cover the cost of the first search, in which case consumers rather do not search at all and the market fails to exist.
} 
the market will not arise when consumer search costs are heterogeneous. Two related papers (Janssen, Moraga-González, and Wildenbeest, 2005; Rauh, 2004) also allow for endogenous consumer participation, although in both papers the type of search cost heterogeneity that is assumed is less general than in our model. Janssen, Moraga-González, and Wildenbeest (2005) employ Stahl's (1989) setting, with the much used "shoppers and non-shoppers" setup. They show that when the extensive search margin plays a role, prices will always increase if the search cost decreases. Our paper shows instead that prices can go both directions and provides conditions under which one or the other occurs. This means that by not restricting the extent of consumer heterogeneity in the market, our model may result in qualitatively very different outcomes than a model that uses the standard shoppers and non-shoppers setup. Moreover, the shoppers and non-shoppers setup does not allow for studying the comparative statics effects of SOSD decreases in search costs, such as those that occurred in the US mutual fund industry. In Rauh (2004) consumer participation is endogenous and proves to be important for the welfare analysis of price ceilings; however, he assumes search costs are uniformly distributed and does not examine the comparative statics effects of higher search costs.

A few recent papers have put forward situations in which lower search costs do not necessarily lead to lower prices, although these findings rely on very different mechanisms. For instance, Chen and Zhang (2011) enrich Stahl's (1989) setting by adding loyal consumers, and show that in such an environment a reduction in the search cost sometimes leads to higher equilibrium prices. In a different framework where search is price-directed, Armstrong and Zhou (2011) show that lower search costs lead to higher prices. A similar result is obtained in Haan and Moraga-González (2011b), who extend the Anderson and Renault (1999) model by making prices observable before search. In a model in which consumers search for various products from multi-product firms, Zhou (2014) demonstrates that product externalities can lead firms to raise their prices when search costs go down. Lastly, in a model with vertical relations, Janssen and Shelegia (2015) encounter situations where retail prices increase as search costs decrease.

Finally, our paper relates to the recent literature on obfuscation, which points out that firms have incentives to obfuscate their products by raising the costs consumers have to incur to inspect their offers (Ellison and Wolitzky, 2012; Wilson, 2010). Our result tells that under certain conditions firms may benefit from doing exactly the opposite, that is, by lowering search costs. 


\section{Sequential search for differentiated products}

Our model builds on the seminal contribution of Wolinsky (1986); specifically we examine a competitive version of his model and we allow consumers to have heterogeneous search costs. ${ }^{5}$ Consider a market with infinitely many consumers and firms and, without loss of generality, normalize the number of consumers per firm to 1 . Firms produce horizontally differentiated products using the same constant returns to scale technology of production; let $r$ be the marginal cost of the firms. Aiming at maximizing their expected profits, firms choose their prices simultaneously. We focus on symmetric Nash equilibria (SNE); let $p^{*}$ denote an SNE price.

A consumer $\ell$ has tastes for a product $i$ described by the following indirect utility function:

$$
u_{i \ell}=\left\{\begin{array}{cl}
\varepsilon_{i \ell}-p_{i} & \text { if she buys product } i \text { at price } p_{i} \\
0 & \text { otherwise }
\end{array}\right.
$$

The parameter $\varepsilon_{i \ell}$ is a match value between consumer $\ell$ and product $i$. We assume that the match value $\varepsilon_{i \ell}$ is the realization of a random variable distributed on the interval $[0, \bar{\varepsilon}]$ according to a cumulative distribution function (CDF) denoted by $F$. Let $f$ be the probability density function (PDF) of $F$. We assume that $f$ is differentiable and that $1-F$ is log-concave. Match values $\varepsilon_{i \ell}$ are independently distributed across consumers and products. Moreover, they are private information of consumers so personalized pricing is not possible. Consumers search sequentially in order to maximize their expected utility. While searching, they have correct beliefs about the equilibrium price. The total cost of search of a consumer $\ell$ who searches $n$ times is $k_{\ell}+n c_{\ell}$, where $k_{\ell}$ is the consumer's fixed cost of search and $c_{\ell}$ indicates her marginal cost of search.

In the following sections we examine the role of search cost heterogeneity in our model. We start with the case in which consumers have heterogeneous marginal search costs. In Section 5 we study what happens when consumers have heterogeneous fixed search costs.

\footnotetext{
${ }^{5}$ In the Appendix we present numerical results showing that the main insights of our paper also hold when the number of firms is finite. With a finite number of firms, however, proving existence and uniqueness of equilibrium is a challenge because the demand of a typical firm consists of a sum of two non-necessarily quasi-concave functions of its own price (see Anderson and Renault, 1999).
} 


\section{Marginal search cost heterogeneity}

Assume that consumers differ in their marginal costs of search. ${ }^{6}$ Specifically, assume that a buyer $\ell$ 's marginal search cost $c_{\ell}$ is drawn independently from a differentiable cumulative distribution function $G$ with support $[\underline{c}, \bar{c}]$. Let $g$ be the density of $G$. We refer to the difference between the upper and lower bound of the search cost distribution as the range of search costs. We require the lower bound of the search cost distribution to be sufficiently low because otherwise no consumer would search and the market would collapse. In this section, we assume that consumers do not need to incur any fixed cost of search; the role of fixed search costs is relegated to Section 5 .

\section{Existence and uniqueness of an SNE price}

We now characterize the SNE price. In order to do so, we derive the payoff of a firm, say $i$, that deviates from the SNE price $p^{*}$ by charging a price $p_{i} \neq p^{*}$. Next, we compute the first order condition (FOC), apply the symmetry condition $p_{i}=p^{*}$, and study the existence and uniqueness of the SNE. For later use, we define the monopoly price as $p^{m}=\arg \max _{p}(p-r)(1-F(p))$.

Consider the (expected) payoff to a firm $i$ that deviates from the equilibrium by charging a price $p_{i}$. In order to compute firm $i$ 's demand, we first need to characterize consumer search behavior. Since consumers do not observe deviations before searching, we can rely on Kohn and Shavell (1974), who study the search problem of a consumer who faces a set of independently and identically distributed options with a known distribution. Kohn and Shavell show that the optimal search rule is static in nature and has the stationary reservation utility property. Accordingly, consider a consumer with search cost $c$ and denote the solution to

$$
h(x) \equiv \int_{x}^{\bar{\varepsilon}}(\varepsilon-x) f(\varepsilon) d \varepsilon=c
$$

in $x$ by $\hat{x}(c)$. The left-hand-side (LHS) of equation (1) is the expected benefit in symmetric equilibrium from searching one more time for a consumer whose best option so far is $x$. Its righthand-side (RHS) is the consumer's cost of search. Hence $\hat{x}(c)$ represents the threshold match value above which a consumer with search cost $c$ will optimally decide not to continue searching for another product. The function $h$ is monotonically decreasing. Moreover, $h(0)=E[\varepsilon]$ and $h(\bar{\varepsilon})=0$. It is readily seen that for any $c \in[\underline{c}, \min \{\bar{c}, E[\varepsilon]\}]$, there exists a unique $\hat{x}(c)$ that

\footnotetext{
${ }^{6}$ Most models in the literature abstract from consumer search cost heterogeneity or only allow for special forms of heterogeneity, typically with some consumers having a search cost equal to zero (usually referred to as the "shoppers"), while the rest faces a positive and identical search cost (the "non-shoppers"). There are some exceptions to this in the literature (e.g., Bénabou, 1993; Rob, 1985; Rauh, 2009; Stahl, 1996; Tappata, 2009) but in these papers search costs are assumed to be sufficiently low so that all consumers search at least once. We will later show that this assumption is restrictive.
} 
solves equation (1). Differentiating equation (1) successively, we obtain

$$
\begin{gathered}
\hat{x}^{\prime}(c)=-\frac{1}{1-F(\hat{x}(c))}<0 \\
\hat{x}^{\prime \prime}(c)=\frac{f(\hat{x}(c))\left[\hat{x}^{\prime}(c)\right]^{2}}{1-F(\hat{x}(c))}>0,
\end{gathered}
$$

which implies that $\hat{x}(c)$ is a decreasing and convex function of $c$ on $[\underline{c}, \min \{\bar{c}, E[\varepsilon]\}]$, with $\hat{x}(E[\varepsilon])=0$ and $\hat{x}(\underline{c}) \leq \bar{\varepsilon}{ }^{7}$

In order to compute firm $i$ 's demand, consider a consumer with search cost $c$ who shows up at firm $i$ to inspect its product after possibly having inspected other products. Let $\varepsilon_{i}-p_{i}$ denote the utility the consumer derives from firm $i$ 's product. Obviously, if alternative $i$ is not the best one so far, the consumer will discard it and search again. Therefore, only when the deal offered by firm $i$ happens to be the best so far, the consumer will consider to stop searching and buy the product right away. For this decision, the consumer compares the gains from an additional search with the costs of such a search. In this comparison, the consumer holds correct expectations about the equilibrium price so she expects the other firms to charge $p^{*}$. The expected gains from searching one more firm, say firm $j$, are equal to $\int_{\varepsilon_{i}-p_{i}+p^{*}}^{\bar{c}}\left[\varepsilon_{j}-\left(\varepsilon_{i}-p_{i}+p^{*}\right)\right] f\left(\varepsilon_{j}\right) d \varepsilon_{j}$. Comparing this to equation (1), it follows that, conditional on having arrived at firm $i$, the probability that buyer $c$ stops searching at firm $i$ is equal to $\operatorname{Pr}\left[\varepsilon_{i}-p_{i}>\hat{x}(c)-p^{*}\right]=1-F\left(\hat{x}(c)+p_{i}-p^{*}\right)$. With the remaining probability, the consumer finds the product of firm $i$ not sufficiently satisfactory and continues searching; with infinitely many firms, such a consumer will surely buy at another firm. Because a consumer with search cost $c$ may visit firm $i$ after having visited no, one, two, three, etc. other firms, the unconditional probability she stops searching and buys at firm $i$ is

$$
\frac{1-F\left(\hat{x}(c)+p_{i}-p^{*}\right)}{1-F(\hat{x}(c))} .
$$

To obtain the payoff of firm $i$ we need to integrate expression (2) over the consumers who decide to search the market for a satisfactory product; in other words, we need to integrate over those consumers who derive expected positive surplus from participation. To compute the surplus a consumer with search cost $c$ obtains from participation, we note that she will stop and buy at the first firm she visits whenever the match value she derives at that firm is greater than $\hat{x}(c)$; otherwise she will drop the first option and continue searching. In the latter case she will encounter herself exactly in the same situation as before because, conditional on participating, she will continue searching until she finds a product satisfactory enough to buy it and leave the

\footnotetext{
${ }^{7}$ Consumers with search $\operatorname{cost} c>E[\varepsilon]$, if any, will automatically drop from the market and can therefore be ignored right away. Therefore $\hat{x}(c)$ is well-defined for every consumer that matters for pricing.
} 
market. Denoting by $C S(c)$ her consumer surplus, we have that: ${ }^{8}$

$$
C S(c)=\hat{x}(c)-p^{*}
$$

Setting this surplus equal to zero, and using equation (1), we obtain the critical search cost value above which consumers will refrain from participating in the market:

$$
\tilde{c}\left(p^{*}\right)=\int_{p^{*}}^{\bar{\varepsilon}}\left(\varepsilon-p^{*}\right) f(\varepsilon) d \varepsilon
$$

Depending on how large the range of search costs is, more or less consumers will choose to search the market for a satisfactory deal. Correspondingly, we define

$$
c_{0}\left(p^{*}\right) \equiv \min \left\{\bar{c}, \tilde{c}\left(p^{*}\right)\right\}
$$

We refer to the decision to search as the extensive search margin. Because $\tilde{c}\left(p^{*}\right)$ is decreasing in $p^{*}$, if consumers expect a higher equilibrium price then fewer of them will choose to search the market for an acceptable product. ${ }^{9}$

The payoff to the deviant firm $i$ is then:

$$
\pi\left(p_{i} ; p^{*}\right)=\left(p_{i}-r\right) D\left(p_{i}, p^{*}\right)
$$

where the demand firm $i$ receives $D\left(p_{i}, p^{*}\right)$ is

$$
D\left(p_{i}, p^{*}\right)=\left\{\begin{array}{cl}
\int_{c}^{c_{0}\left(p^{*}\right)} \frac{1-F\left(\hat{x}(c)+p_{i}-p^{*}\right)}{1-F(\hat{x}(c))} g(c) d c & \text { if } p_{i}<p^{*} \\
\int_{\max \left\{\underline{c}, \hat{c}\left(p_{i}\right)\right\}}^{c_{0}\left(p^{*}\right)} \frac{1-F\left(\hat{x}(c)+p_{i}-p^{*}\right)}{1-F(\hat{x}(c))} g(c) d c & \text { if } p_{i} \geq p^{*}
\end{array}\right.
$$

where $\hat{c}\left(p_{i}\right)$ is the solution of the equation $\hat{x}(c)+p_{i}-p^{*}=\bar{\varepsilon}$ in $c$.

The FOC is given by (for $\left.p_{i}<p^{*}\right)$ :

$$
\int_{\underline{c}}^{c_{0}\left(p^{*}\right)} \frac{1-F\left(\hat{x}(c)+p_{i}-p^{*}\right)}{1-F(\hat{x}(c))} g(c) d c-\left(p_{i}-r\right) \int_{\underline{c}}^{c_{0}\left(p^{*}\right)} \frac{f\left(\hat{x}(c)+p_{i}-p^{*}\right)}{1-F(\hat{x}(c))} g(c) d c=0 .
$$

Applying symmetry, i.e., $p_{i}=p^{*}$, we can rewrite the FOC as: ${ }^{10}$

$$
p^{*}=r+\frac{G\left(c_{0}\left(p^{*}\right)\right)}{\int_{\underline{c}}^{c_{0}\left(p^{*}\right)} \frac{f(\widehat{x}(c))}{1-F(\hat{x}(c))} g(c) d c} .
$$

\footnotetext{
${ }^{8}$ For a formal derivation of this expression, see the Appendix.

${ }^{9}$ The standard assumption in the search cost literature has been that $c_{0}\left(p^{*}\right)=\bar{c}$, which implies that all consumers search at least once. This kind of fully-covered market assumption (in the sense that all consumers search) is clearly restrictive and, as we will show later, it can lead to wrong conclusions about the effects of higher search costs on equilibrium prices.

${ }^{10}$ Note that the first-order necessary condition for a symmetric equilibrium is the same when we assume that the deviating price $p \geq p^{*}$.
} 
We now show that a candidate symmetric equilibrium price exists and is unique. First consider the case in which search costs are sufficiently low, i.e. $\bar{c}<\tilde{c}\left(p^{*}\right)$, hence $G\left(c_{0}\left(p^{*}\right)\right)=1$. In terms of exogenous parameters, this occurs under the following restriction:

\section{Condition 1.}

$$
\bar{c} \leq \int_{r+\frac{1}{\int_{\underline{c}}^{\bar{c}} \frac{f(\widehat{x}(c))}{1-F(\bar{x}(c))} g(c) d c}}^{\bar{\varepsilon}}\left(\varepsilon-r-\frac{1}{\int_{\underline{c}}^{\bar{c}} \frac{f(\widehat{x}(\widehat{1}))}{1-F(\widehat{x}(c))} g(c) d c}\right) f(\varepsilon) d \varepsilon .
$$

Because under Condition $1 c_{0}\left(p^{*}\right)=\bar{c}$, the expression (6) gives the candidate equilibrium price explicitly. In this case, obviously, there exists a unique candidate equilibrium price.

When search costs are not restricted to be sufficiently small and Condition 1 is violated then $\bar{c}>\tilde{c}\left(p^{*}\right)$ and correspondingly $G\left(c_{0}\left(p^{*}\right)\right)<1$. In this case the equilibrium price is given implicitly by the solution to equation (6). We now show that equation (6) has a unique solution in this situation as well. For this we define the function

$$
L(p) \equiv G(\tilde{c}(p))-(p-r) \int_{\underline{c}}^{\tilde{c}(p)} \frac{f(\widehat{x}(c))}{1-F(\widehat{x}(c))} g(c) d c
$$

for $p \in\left[r, p^{m}\right]$, where $p^{m}$ as before denotes the monopoly price. Note that

$$
L(r)=G(\tilde{c}(r))>0 .
$$

Also observe that $L\left(p^{m}\right)$ can be written as

$$
L\left(p^{m}\right)=\int_{\underline{c}}^{\tilde{c}\left(p^{m}\right)} \frac{1-F(\hat{x}(c))-\left(p^{m}-r\right) f(\hat{x}(c))}{1-F(\hat{x}(c))} g(c) d c .
$$

The sign of this expression depends on the sign of the numerator of the fraction in the integrand. We now argue that $L\left(p^{m}\right)<0$ because $1-F(\hat{x}(c))-\left(p^{m}-r\right) f(\hat{x}(c)) \leq 0$ for all $c \in\left[\underline{c}, \tilde{c}\left(p^{m}\right)\right]$. In fact, note that by $\log$-concavity of $f$, because $\hat{x}(c)$ decreases in $c$, it follows that $f(\hat{x}(c)) /[1-F(\hat{x}(c))]$ decreases in $c$, which implies that $1-F(\hat{x}(c))-\left(p^{m}-r\right) f(\hat{x}(c))$ increases in $c$. Because $\hat{x}(c(p))=p$, if we set $c=\tilde{c}\left(p^{m}\right)$ in the expression $1-F(\hat{x}(c))-\left(p^{m}-r\right) f(\hat{x}(c))$, we get the monopoly pricing rule $1-F\left(p^{m}\right)-\left(p^{m}-r\right) f\left(p^{m}\right)=0$. We can now conclude that $L\left(p^{m}\right)<0$ because the expression $1-F(\hat{x}(c))-\left(p^{m}-r\right) f(\hat{x}(c))$ is increasing in $c$ and takes on value zero when we compute it at the upper bound of the integral.

Taken together, $L(r)>0$ and $L\left(p^{m}\right)<0$ imply that a candidate equilibrium price $p^{*} \in$ $\left[r, p^{m}\right]$ exists. We finally note that

$$
\begin{aligned}
\frac{d L(p)}{d p} & =g(\tilde{c}(p)) \frac{d \tilde{c}(p)}{d p}-(p-r) f(p) g(\tilde{c}(p)) \frac{d \tilde{c}(p)}{d p}-\int_{\underline{c}}^{\tilde{c}(p)} f(\hat{x}(c)) g(c) d c \\
& =\frac{1-F(p)-(p-r) f(p)}{1-F(p)} g(\tilde{c}(p)) \frac{d \tilde{c}(p)}{d p}-\int_{\underline{c}}^{\tilde{c}(p)} f(\hat{x}(c)) g(c) d c
\end{aligned}
$$


is negative for any $p \in\left[r, p^{m}\right]$, which implies that the candidate equilibrium price is unique. This follows from the fact that $1-F(p)-(p-r) f(p) \geq 0$ (because it is the first order derivative of the monopoly payoff $(p-r)(1-F(p))$, which is log-concave) and $d \tilde{c}(p) / d p<0$ (because, from equation (3), $\tilde{c}$ is decreasing in $p$ ). In particular, at the candidate equilibrium price $p^{*}$ we must have $d L\left(p^{*}\right) / d p<0$.

Our next result provides conditions for existence of a symmetric equilibrium.

Theorem 1. There may be two types of SNE in the model of sequential search for differentiated products with heterogeneous marginal costs of search:

(A) An SNE where all consumers conduct at least a first search, in which case Condition 1 holds so that $\bar{c} \leq \tilde{c}\left(p^{*}\right)$. In this type of equilibrium firms charge a price given by expression (6) after setting $c_{0}\left(p^{*}\right)=\bar{c}$, and where $\hat{x}(c)$ solves equation (1).

(B) An SNE where only some of the consumers search for a satisfactory product, in which case Condition 1 is violated so that $\bar{c}>\tilde{c}\left(p^{*}\right)$. In this type of equilibrium firms charge a price given by expression (6) after setting $c_{0}\left(p^{*}\right)=\int_{p^{*}}^{\bar{\varepsilon}}\left(\varepsilon-p^{*}\right) f(\varepsilon) d \varepsilon$; moreover, the fraction of consumers $G\left(\int_{p^{*}}^{\bar{\varepsilon}}\left(\varepsilon-p^{*}\right) f(\varepsilon) d \varepsilon\right)$ conducts at least a first search while the rest of the consumers do not search at all and leave the market.

Let $\hat{x}^{-1}(t)$ denote the inverse function of $\hat{x}(c)$. When the function $g\left(\hat{x}^{-1}(t)\right)$ is log-concave in $t$, then the SNE exists and is unique.

The proof, whose details are in the Appendix, is as follows. Because a direct verification of the second order conditions does not deliver clear-cut results, we proceed by showing that the demand function of an individual firm is a log-concave function of its own price under the assumption that $g\left(\hat{x}^{-1}(t)\right)$ is log-concave in $t$. Once this is proven, we know that the firm profit function (4) is quasi-concave in its own price so that the unique candidate equilibrium price given by expression (6) is indeed an equilibrium. In order to prove that the demand function of a firm is log-concave in the firm's own price, we first show that the demand from a single consumer is log-concave both in price and in consumer reservation values; after this we make use of Theorem 6 in Prékopa (1973), which shows that integration over consumer reservation values preserves log-concavity.

\section{The comparative statics effects of lower search costs}

We now proceed to study the impact of lower marginal search costs on the equilibrium prices given by Theorem 1. In order to address this question, we parametrize the CDF of search 
costs by a positive parameter $\beta$ that shifts the distribution. Specifically, let $G(c ; \beta)$ denote the parametrized CDF of search costs; let $g(c ; \beta)$ denote the parametrized PDF of search costs with support $[\underline{c}(\beta), \bar{c}(\beta)]^{11}$

We study FOSD and SOSD decreases in search costs. Concretely, we say that search costs decrease in the sense of FOSD whenever a decrease in $\beta$ implies an increase in the search cost distribution for all $c$, i.e., when $\partial G(c ; \beta) / \partial \beta \leq 0$ for all $c$ (and $<0$ for some $c$ ). Alternatively, we say that search costs decrease in the sense of SOSD whenever

$$
\int_{\underline{c}(\beta)}^{c} \frac{\partial G(x ; \beta)}{\partial \beta} d x \leq 0 \text { for all } c(\text { and }<0 \text { for some } c) .
$$

Note that SOSD is implied by FOSD (not vice-versa).

Let $p^{*}(\beta)$ be the corresponding SNE price for a given $\beta$. In order to develop some intuition, it is useful to recall that, as usual with monopolistic competition, we can write the equilibrium price as:

$$
p^{*}(\beta)=\frac{r}{1-\frac{1}{\epsilon\left(p^{*} ; \beta\right)}}
$$

where

$$
\epsilon\left(p^{*} ; \beta\right) \equiv-\frac{p^{*} D^{\prime}\left(p^{*} ; \beta\right)}{D\left(p^{*} ; \beta\right)}
$$

represents the price elasticity of demand. We are interested in the behavior of $p^{*}(\beta)$ with respect to $\beta$.

\subsection{Low search costs}

Consider first the case of Theorem 1A. When the upper bound of the search cost distribution is sufficiently low and all consumers search, a change in search costs only affects the intensive search margin. The equilibrium price is equal to

$$
p^{*}(\beta)=r+\frac{1}{\int_{\underline{c}(\beta)}^{\bar{c}(\beta)} \frac{f(\widehat{x}(c))}{1-F(\hat{x}(c))} g(c ; \beta) d c} .
$$

Note that the denominator of the fraction on the RHS of equation (10) is nothing else than the expectation of the hazard rate $f(\hat{x}(c)) /(1-F(\hat{x}(c)))$. By log-concavity of $1-F$, note that the mentioned hazard rate increases in $\hat{x}(c)$ and decreases in $c$.

From Theorem 1 in Hadar and Russell (1971), a FOSD decrease in search costs then immediately implies that the expected value of the hazard rate goes up. It follows, then, that

\footnotetext{
${ }^{11}$ To be as general as possible we allow the support of the density of search costs to depend on $\beta$. We come back to this issue later.
} 
the equilibrium price (10) unambiguously decreases when $\beta$ decreases. The intuition is straightforward: when search costs go down, consumers' reservation values rise; this increases search activity and demand becomes more elastic. In fact, when all consumers search $D\left(p^{*} ; \beta\right)=1$ and the elasticity of demand simply equals

$$
\epsilon\left(p^{*} ; \beta\right)=p^{*} \int_{\underline{c}(\beta)}^{\bar{c}(\beta)} \frac{f(\hat{x}(c))}{1-F(\hat{x}(c))} g(c, \beta) d c .
$$

As we have argued above, a fall in search costs increases the value of the integral in equation (11) and thereby the elasticity of demand goes up. The equilibrium price must then decrease.

With additional conditions on the match value density we can state what happens when search costs decrease in the sense of SOSD (see Theorem 2 of Hadar and Russell, 1971).

Proposition 1. Let $G(c ; \beta)$ be a parametrized search cost $C D F$ with positive density on $[\underline{c}(\beta), \bar{c}(\beta)]$. Then:

(A) An FOSD decrease in search costs results in a fall in the equilibrium price given by Theorem $1 A$.

(B) An SOSD decrease in search costs results in a fall in the equilibrium price given by Theorem $1 A$ provided that $f^{\prime \prime}[1-F]^{2}+4 f f^{\prime}[1-F]+3 f^{3} \geq 0$ (which for example holds when $f$ is uniform).

The proof of Proposition 1B can be found in the Appendix.

\subsection{Sufficiently high search costs}

We now move to the case of Theorem 1B. In this case not all consumers choose to search so a change in the distribution of search costs affects both the intensive and the extensive search margins. The equilibrium price is given by the solution to equation

$$
L\left(p^{*} ; \beta\right) \equiv G\left(\tilde{c}\left(p^{*}\right) ; \beta\right)-\left(p^{*}-r\right) \int_{\underline{c}(\beta)}^{\tilde{c}\left(p^{*}\right)} \frac{f(\hat{x}(c))}{1-F(\hat{x}(c))} g(c, \beta) d c=0 .
$$

It turns out to be quite useful to rewrite this, dividing by $G\left(\tilde{c}\left(p^{*}\right) ; \beta\right)$, as follows:

$$
\tilde{L}(p ; \beta) \equiv 1-\left(p^{*}-r\right) \int_{\underline{c}(\beta)}^{\tilde{c}\left(p^{*}\right)} \frac{f(\hat{x}(c))}{1-F(\hat{x}(c))} \tilde{g}(c, \beta) d c=0,
$$

where

$$
\tilde{g}\left(c, \beta ; \tilde{c}\left(p^{*}\right)\right) \equiv \frac{g(c, \beta)}{G\left(\tilde{c}\left(p^{*}\right) ; \beta\right)}
$$

denotes the search cost density truncated from above by $\tilde{c}\left(p^{*}\right)$. 
By the implicit function theorem, the impact of a (small) decrease in $\beta$ on the equilibrium price in equation (12) is given by the sign of

$$
\frac{d p^{*}(\beta)}{d \beta}=-\frac{\frac{\partial \tilde{L}\left(p^{*} ; \beta\right)}{\partial \beta}}{\frac{\partial \tilde{L}\left(p^{*} ; \beta\right)}{\partial p^{*}}} .
$$

We have already argued above that the function $L\left(p^{*} ; \beta\right)$ is decreasing in $p^{*}$, and so is $\tilde{L}\left(p^{*} ; \beta\right)$, so the denominator of equation (13) is negative. Therefore the sign of $d p^{*}(\beta) / d \beta$ depends on the sign of $\partial \tilde{L}(\cdot) / \partial \beta$.

Note that equation (12) is very similar to equation (10). In fact, the only difference is that the integral in equation (12) is the expected value of the hazard rate of the consumers who choose to search, and that is why the integral involves the truncated density. Therefore, the answer to the question what happens to the price when search costs go down boils down to finding out what happens to the mentioned expected value.

Denoting the distribution of search costs truncated above by $\tilde{c}\left(p^{*}\right)$ by

$$
\left.\tilde{G}\left(c, \beta ; \tilde{c}\left(p^{*}\right)\right) \equiv \int_{\underline{c}(\beta)}^{c} \tilde{g}\left(x, \beta ; \tilde{c}\left(p^{*}\right)\right)\right) d x,
$$

we can state directly (and without proof) the following extension of Proposition 1.

Proposition 2. Let $G(c ; \beta)$ be a parametrized search cost $C D F$ with positive density on $[\underline{c}(\beta), \bar{c}(\beta)]$. Then:

(A) An FOSD decrease in search costs results in a fall (increase) in the equilibrium price given by Theorem $1 B$ if $\partial \tilde{G}(c, \beta) / \partial \beta \leq(\geq) 0$ for all $c$ (and $<(>) 0$ for some $c$ ).

(B) Assume that $f^{\prime \prime}[1-F]^{2}+4 f f^{\prime}[1-F]+3 f^{3} \geq 0$. Then an SOSD decrease in search costs results in a fall (increase) in the equilibrium price given by Theorem $1 B$ if

$$
\left.\int_{\underline{c}(\beta)}^{c} \frac{\partial \tilde{G}\left(x ; \beta, \tilde{c}\left(p^{*}\right)\right)}{\partial \beta} d x \leq 0 \text { for all } c \text { (and }<0 \text { for some } c\right) \text {. }
$$

This result emphasizes that what really matters for the relationship between the equilibrium price and $\beta$ is the way in which the (truncated) search cost distribution of the participating consumers is affected by changes in $\beta$. If a FOSD decrease in search costs results in a FOSD decrease in the distribution of search costs conditional on participation, then the equilibrium price will surely decrease. Otherwise, the equilibrium price will increase. This result is similar for SOSD decreases in search costs.

The intuition behind this result is as follows. When not all consumers search the elasticity of demand is

$$
\epsilon\left(p^{*} ; \beta\right)=p^{*} \int_{\underline{c}(\beta)}^{\tilde{c}\left(p^{*}\right)} \frac{f(\hat{x}(c))}{1-F(\hat{x}(c))} \tilde{g}(c, \beta) d c
$$


A fall in search costs makes consumers more picky and they search more. Because of this effect, firms have an incentive to lower their prices. However, a fall in search costs brings new consumers into the market. These consumers turn out to have relatively large search costs because otherwise they would have been searching previously. Because of this effect, firms have an incentive to raise their prices. If the second effect is weak and the distribution of search costs conditional on search has lower search costs then the price will fall. If the second effect is sufficiently strong, then the price will rise.

The question is then, under which conditions a (FOSD or SOSD) decrease in search costs results in a (FOSD or SOSD) decrease in the truncated distribution of search costs? We now present a well-known result from the statistics literature that enables us to address this question: ${ }^{12}$

Lemma 1. [Ahlswede and Daykin, 1978]. Let $f_{1}, f_{2}, f_{3}, f_{4}$ be non-negative functions on $[a, b]$ such that $f_{1}(x) \cdot f_{2}(y) \leq f_{3}(\max \{x, y\}) \cdot f_{4}(\min \{x, y\})$ for all $x, y \in[a, b]$. Then

$$
\int_{a}^{b} f_{1}(x) d x \cdot \int_{a}^{b} f_{2}(x) d x \leq \int_{a}^{b} f_{3}(x) d x \cdot \int_{a}^{b} f_{4}(x) d x .
$$

Athey (2002) uses this result to show that log-supermodularity is preserved by integration. Our use of this Lemma is different; as far as we know, it has not been used before to prove the following result.

Proposition 3. Assume that $g(c ; \beta)$ has the increasing (decreasing) likelihood ratio property, that is,

$$
g(c ; \gamma) g(d ; \beta) \leq(\geq) g(c ; \beta) g(d ; \gamma)
$$

for any $c \leq d$ and $\beta \leq \gamma$. Then, the distribution of search costs truncated from above by $\tilde{c}\left(p^{*}\right)$ corresponding to $\beta(\gamma)$, first-order stochastically dominates the distribution of search cost truncated from above by $\tilde{c}\left(p^{*}\right)$ corresponding to $\gamma(\beta)$, that is,

$$
G\left(c ; \beta \mid c \leq \tilde{c}\left(p^{*}\right)\right) \geq(\leq) G\left(c ; \gamma \mid c \leq \tilde{c}\left(p^{*}\right)\right)
$$

for all $c$ and $\tilde{c}\left(p^{*}\right)$.

The proof of this result is in the Appendix. Note that the ILRP (DLRP) is equivalent to log-supermodularity (log-submodularity) (cf. Athey, 2002).

By combining Propositions 2 and 3 we can state sufficient conditions under which a reduction in search costs will decrease or increase the equilibrium price. Namely, when the search cost

\footnotetext{
${ }^{12}$ We are indebted to Paulo Monteiro for alerting us about this result.
} 
density has the ILRP with respect to parameter $\beta$, then a decrease in $\beta$ will cause a FOSD decrease of the truncated search cost distribution, thereby leading to a price decrease. By contrast, when the search cost density has the DLRP, a decrease in $\beta$ will cause a FOSD increase of the truncated search cost distribution and this will result in a higher price.

The final observation we make is that we need to ensure that ILRP and DLRP are compatible with FOSD and SOSD. Obviously, ILRP is compatible with FOSD (and SOSD by implication) because the latter is implied by the former. For DLRP to be compatible with FOSD (and hence SOSD) we just need the (upper bound of the) support of search cost to increase with $\beta$ (below we present a few examples).

To summarize, combining Propositions 1-3, we have obtained the following comparative statics results.

Theorem 2. Let $G(c ; \beta)$ be a parametrized search cost $C D F$ with positive density on $[\underline{c}(\beta), \bar{c}(\beta)]$. Then:

(A) If a decrease in $\beta$ signifies a FOSD decrease of search costs, then the equilibrium price in Theorem $1 A$ will decrease. If it signifies a SOSD decrease in search costs then the price will decrease provided that $f^{\prime \prime}[1-F]^{2}+4 f f^{\prime}[1-F]+3 f^{3} \geq 0$.

(B) If a decrease in $\beta$ signifies a FOSD or a SOSD decrease in search costs and if $g(c ; \beta)$ has the ILRP with respect to $\beta$, then the equilibrium price in Theorem $1 B$ will decrease. If a decrease in $\beta$ signifies a FOSD or a SOSD decrease in search costs and if $g(c ; \beta)$ has the decreasing likelihood ratio property with respect to $\beta$ (in which case we must have $d \bar{c}(\beta) / d \beta>0$ ), then the equilibrium price in Theorem $1 B$ will increase.

The intuition behind the role played by the increasing or decreasing likelihood ratio property of the search cost density in Theorem 2 can better be conveyed by means of a couple of instructive examples. Suppose that search costs are distributed on a fixed interval and that the density is decreasing. Moreover, suppose that the search cost density satisfies the ILRP, that is, for $\gamma \geq \beta$, the relative frequency $g(c ; \gamma) / g(c ; \beta)$ is increasing in $c$. The ILRP implies that $g(c ; \beta)$ must decrease more rapidly than $g(c ; \gamma)$. Because both are densities and their integrals must equal 1 , it must be the case that $g(c ; \beta)$ is higher than $g(c ; \gamma)$ for low search costs and otherwise for high search costs. This in turn implies that for any truncation point, the average consumer under $g(c ; \beta)$ has a lower search cost than that under $g(c ; \gamma)$. For densities with the ILRP, a decrease in search costs has a relatively stronger effect on the intensive search margin than on the extensive search margin and this causes the average consumer to become less elastic after search costs go down. This leads to a decrease in the equilibrium price. A similar argument 
can be made for increasing densities. In that case, $g(c ; \beta)$ must increase less rapidly than $g(c ; \gamma)$ which implies that the frequency of low search cost consumers under $g(c ; \beta)$ is higher than under $g(c ; \gamma)$. An example, which is further elaborated in the Appendix, follows.

Example 1: ILRP Assume that match values are distributed on $[0,1]$ according to the uniform distribution and that search costs are distributed on $[0, \beta]$ according to the density function

$$
g(c)=\frac{\beta(2+\delta)-2 c}{\beta^{2}(1+\delta)}, \text { with } \delta \geq 0 .
$$

The CDF of $g$ is

$$
G(c)=\frac{c(\beta(2+\delta)-c)}{\beta^{2}(1+\delta)}
$$

and note that an increase in the parameters $\beta$ or $\delta$ signify a FOSD shift of the search cost CDF. When $\beta$ changes, the support changes but when $\delta$ changes, the support remains the same. Moreover, we observe that $g$ has the ILRP with respect to parameters $\beta$ and $\delta$. For this example, we can then state that an equilibrium exists and is unique and that an increase in either $\beta$ or $\delta$ increases the equilibrium price given by Theorem $1 A$ and increases the equilibrium price given by Theorem $1 B$.

The effect of lower search costs when the extensive margin plays a role is illustrated in Figure 1. The red curve in graph 1 (a) is the density of search costs for $\delta=2$; the blue curve for $\delta=1$. In this graph we set $\beta=1$. The move from the red to the blue density represents a FOSD decrease of search costs, as can be seen in graph 1(c). The vertical dashed line indicates the threshold search cost above which consumer choose not to search the market for a satisfactory product. The corresponding truncated densities and distributions are given in graphs 1(b) and $1(\mathrm{~d})$. What we see is that a FOSD decrease of search costs translates into a FOSD decrease of the search costs of participating consumers.

With densities that satisfy the DLRP, $g(c ; \gamma) / g(c ; \beta)$ is decreasing in $c$ and we correspondingly have the opposite. The following example illustrates the situation when the search cost density satisfies the DLRP.

Example 2: DLRP Assume that match values are distributed on $[0,1]$ according to the uniform distribution and that the search cost density is

$$
g(c)=\frac{1+\delta}{\beta(2+\delta)}\left[1+\left(\frac{c}{\beta}\right)^{\delta}\right], \text { with } 0<\delta \leq 1 / 2 .
$$

The CDF of $g$ is

$$
G(c)=\frac{c}{\beta(2+\delta)}\left[1+\delta+\left(\frac{c}{\beta}\right)^{\delta}\right]
$$




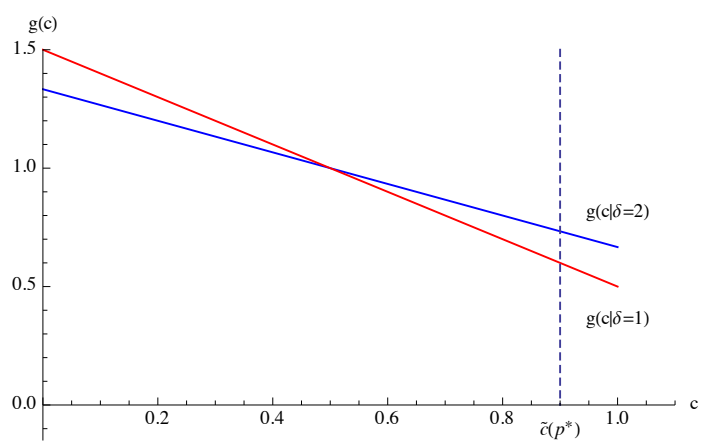

(a) Densities

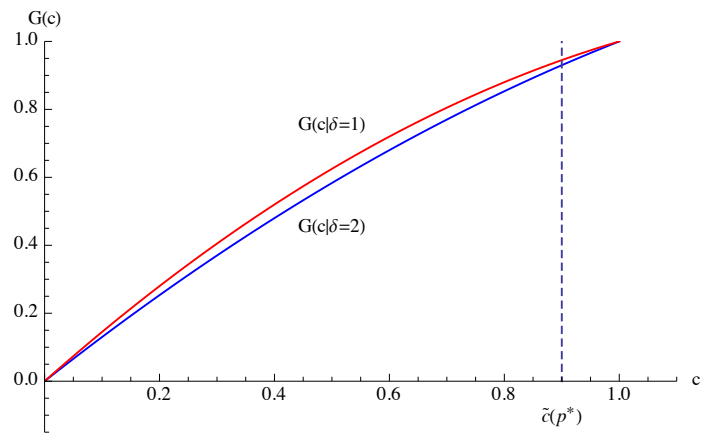

(c) Distributions

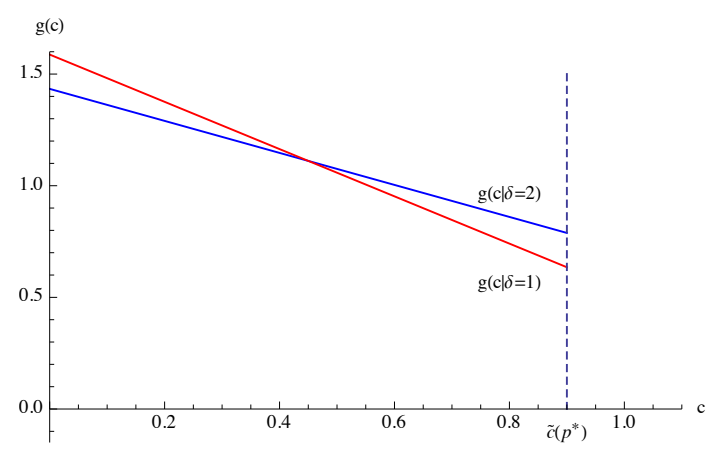

(b) Truncated densities

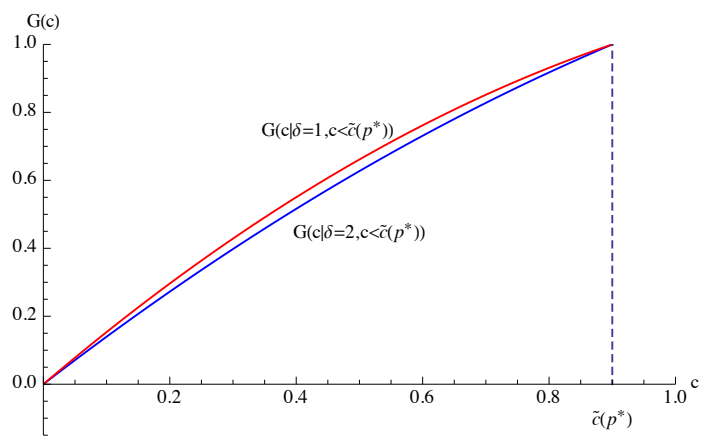

(d) Truncated distributions

Figure 1: ILRP and the effect of higher search costs on (truncated) densities and distributions.

and note that an increase in parameter $\beta$ implies a FOSD shift of the search cost CDF. Moreover, $g$ has the DLRP with respect to parameter $\beta$. (For DLRP to be consistent with FOSD, as mentioned in Theorem 2, the support has to vary when we move $\beta$, which is the case here.) For this example we can then state that an equilibrium exists and is unique and that a decrease in search costs decreases the equilibrium price given by Theorem $1 \mathrm{~A}$ but increases the equilibrium price given by Proposition $1 B$.

Example 2 is illustrated in Figure 2. The blue curves represent the densities and distributions, as well as the truncated counterparts when $\beta=1.5$. The red curves show the case of $\beta=1$. The parameter $\delta=1 / 2$. The move from the blue curves to the red ones represent a FOSD fall in search costs, as can be seen in graph 2(c). What we can see is that a decrease in search cost results in a pool of consumers participating that have higher search costs than before (graphs $2(\mathrm{~b})$ and 2(d)). As a result, prices will increase as search costs fall.

\section{Fixed search cost heterogeneity}

Sometimes consumers also face a fixed search cost before their actual search begins, for instance when they first have to start up a computer, log onto a website, or drive to a shopping mall. 


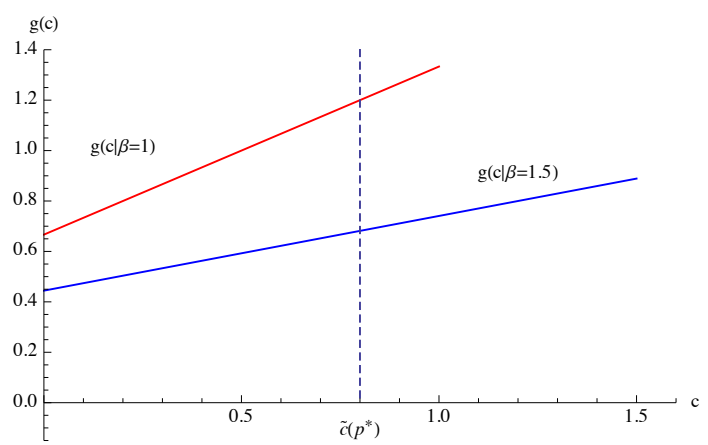

(a) Densities

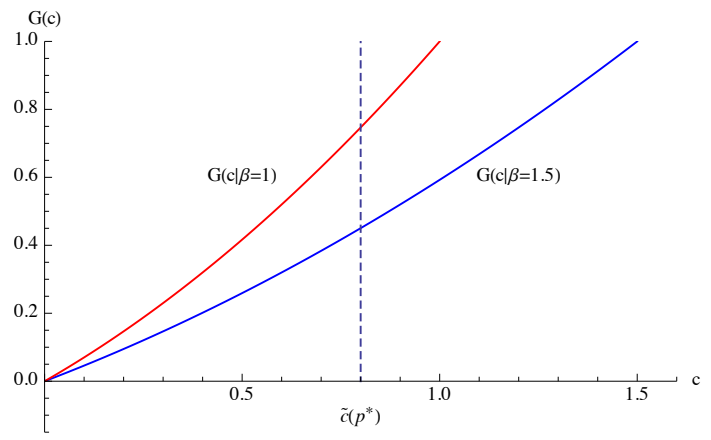

(c) Distributions

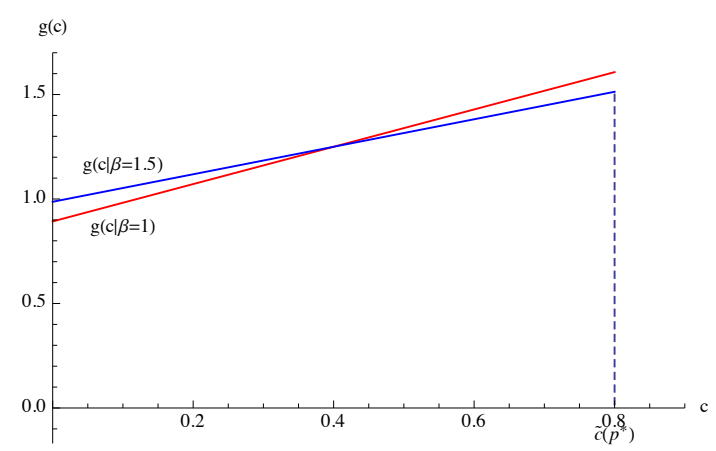

(b) Truncated densities

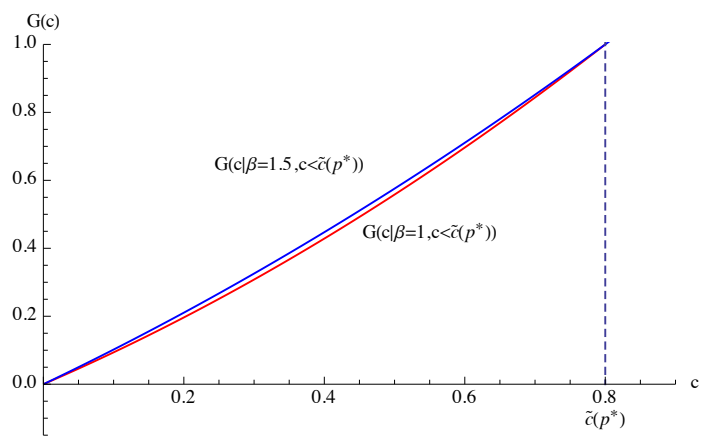

(d) Truncated distributions

Figure 2: DLRP and the effect of higher search costs on (truncated) densities and distributions.

In this section, we study the effect of an increase in fixed search costs on equilibrium prices. The main objective is to show that because fixed costs do not have a bearing on the gains from search, increases in fixed search costs, even if they result in a fall in demand, do not have an impact on the equilibrium price.

Suppose that consumers have to pay a fixed search cost upfront, denoted $k$, in order to start searching. Suppose further that consumers are heterogeneous in their fixed search costs. Let $\mathcal{K}(k)$ be the distribution of fixed search costs, with support $[\underline{k}, \bar{k}]$. After paying this cost, consumers proceed as in the model of Section 2, and face a common marginal search cost equal to $c$.

Since fixed search costs are sunk after the first attempt to find a satisfactory product, the search rule given by the solution to equation (1) remains the same. Correspondingly, the surplus of a consumer with fixed search cost $k$ is equal to $C S(k)=\hat{x}(c)-p^{*}-k$. For a consumer to enter the market this surplus must be strictly positive. Setting $C S(k)=0$ and solving for $k$ gives a critical fixed search cost, denoted $\tilde{k}\left(p^{*}\right)$, above which consumers will abstain from searching for an acceptable product. Defining $k_{0}\left(p^{*}\right) \equiv \min \left\{\bar{k}, \tilde{k}\left(p^{*}\right)\right\}$ the payoff analogous to the payoff of 
the deviant firm $i$ in equation (4) is given by

$$
\pi\left(p_{i} ; p^{*}\right)=\left(p_{i}-r\right) \int_{\underline{k}}^{k_{0}\left(p^{*}\right)} \frac{1-F\left(\hat{x}(c)+p_{i}-p^{*}\right)}{1-F(\hat{x}(c))} d \mathcal{K} \text { for } p_{i}<\bar{\varepsilon}-\hat{x}(c)+p^{*}
$$

and zero otherwise. Because fixed search costs do not affect the reservation value $\hat{x}(c)$, by integrating we obtain

$$
\pi\left(p_{i} ; p^{*}\right)=\mathcal{K}\left(\tilde{k}_{0}\left(p^{*}\right)\right)\left(p_{i}-r\right) \frac{1-F\left(\hat{x}(c)+p_{i}-p^{*}\right)}{1-F(\hat{x}(c))} .
$$

Inspection of equation (15) immediately reveals that a change in the distribution of the fixed search costs has no bearing on equilibrium pricing because, even though it affects demand, it does it in a way that does not modify the elasticity of demand.

The main distinction between heterogeneity in the fixed search cost and heterogeneity in the marginal search cost is that composition-of-demand effects do not matter for pricing in the former case, but do matter in the latter case. Because the marginal benefits from search for the consumers who choose to search are not affected by an increase in fixed search costs, the elasticity of demand is constant no matter the pool of consumers who choose to search. With heterogeneity in the marginal costs of search, this is quite different because the elasticity of demand intimately depends on the pool of consumers who choose to search. We conclude by emphasizing that the mechanism we have identified in this paper does not just arise for any change in the composition of demand. In fact, for the elasticity of average demand to increase or decrease as search costs increase, consumers' marginal benefits from search need to change as well.

\section{Conclusions}

Most existing consumer search models are analyzed under the assumption that all consumers search at least once in equilibrium. In this paper we have argued that it is hard to reconcile this type of "fully-covered-market" assumption with the general idea that distinct consumers have different search costs and that, in many markets, some consumers may simply find it not worthy to start searching for a satisfactory product. Since the decision to search depends on the equilibrium price, the existing literature has neglected an important role of the price mechanism, namely, that prices ought to affect the share of consumers who choose to search for a product in the first place.

We have studied how prices are determined in a model of search for differentiated products while allowing for arbitrary search cost heterogeneity. Our first contribution has been to provide 
results on the existence and uniqueness of equilibrium. We have shown that when the search cost density is sufficiently log-concave, a symmetric Nash equilibrium exists and is unique. In proving this result, we have exploited a mathematical result due to Prékopa (1973) which shows that log-concavity is preserved by integration.

We have also revisited the question how a decrease in search costs affects the level of prices. A decrease in search costs affects not only how much consumers search but also who chooses to search. Recognizing that the price mechanism affects the search composition of demand turns out to be critical for understanding how prices and profits are affected by lower search costs. When search costs decrease there are two effects operating in opposite directions: the average consumer searches more but the identity of the average consumer changes because of the inflow of consumers with relatively high search costs. We have referred to the first effect as the effect on the intensive search margin and to the second one as the effect on the extensive search margin.

Our second contribution has been on identifying sufficient and intuitive conditions on search cost densities under which a decrease in search costs in the sense of FOSD or SOSD has an unambiguous effect on the equilibrium price. We have singled out a critical property of search cost densities that plays a decisive role, namely, log-supermodularity (or monotone increasing likelihood ratio property). When the search cost density is log-supermodular, a decrease in search costs has a relatively stronger impact on the extensive search margin than on the intensive search margin. The intuition is that with log-supermodular densities, a decrease in search costs increases the relative frequency of consumers with low search costs. As a result, the average consumer who chooses to search has a lower search cost and is correspondingly more elastic. This causes the equilibrium price to fall. By contrast, when the search cost density is log-submodular (or has the monotone decreasing likelihood ratio property), a decrease in search frictions raises the relative frequency of consumers with high search costs. Correspondingly, the average (over the consumers who continue choosing to search for an acceptable product) demand becomes less elastic and thereby prices increase. This result is in line with Hortaçsu and Syverson's (2004) empirical observation that prices went up in the US mutual fund industry during the late nineties in spite of the SOSD decrease in search costs that they estimate. 


\section{APPENDIX}

Derivation of consumer surplus. As mentioned in the main text, a consumer with search cost $c$ will stop and buy after the first search when $\varepsilon>\hat{x}(c)$; otherwise she will drop the first option and continue searching, in which case she will encounter herself exactly in the same situation as before because, conditional on participating, the consumer will continue searching until she finds a match value for which it is worth to stop searching. Denoting by $C S(c)$ her consumer surplus, recursively, we must have:

$$
C S(c)=-c+(1-F(\hat{x}(c))) \frac{\int_{\hat{x}(c)}^{\bar{\varepsilon}}\left(\varepsilon-p^{*}\right) f(\varepsilon) d \varepsilon}{1-F(\hat{x}(c))}+F(\hat{x}(c)) C S(c) .
$$

Solving for $C S(c)$ gives

$$
C S(c)=\frac{\int_{\hat{x}(c)}^{\bar{\varepsilon}}\left(\varepsilon-p^{*}\right) f(\varepsilon) d \varepsilon-c}{1-F(\hat{x}(c))} .
$$

Using the value of $c$ from equation (1) we obtain

$$
C S(c)=\frac{\int_{\hat{x}(c)}^{\bar{\varepsilon}}\left(\varepsilon-p^{*}\right) f(\varepsilon) d \varepsilon-\int_{\hat{x}(c)}^{\bar{\varepsilon}}(\varepsilon-\hat{x}(c)) f(\varepsilon) d \varepsilon}{1-F(\hat{x}(c))}=\frac{\int_{\hat{x}(c)}^{\bar{\varepsilon}}\left(\hat{x}(c)-p^{*}\right) f(\varepsilon) d \varepsilon}{1-F(\hat{x}(c))}=\hat{x}(c)-p^{*},
$$

which is the expression we give in the text.

Proof of Theorem 1. It remains to prove that the equilibrium exists when $g\left(\hat{x}^{-1}(t)\right)$ is $\log$-concave in $t$. For this, we prove that the demand function of a firm $i$ in equation (16) is $\log$-concave in its own price $p_{i}$. Once this is proven, it follows that the firm profit function (4) is quasi-concave in its own price so that the unique candidate equilibrium price given by equation (6) is indeed an equilibrium.

We start by rewriting the demand function in a more convenient way. Let us use the following change of variables: $t=\hat{x}(c)$ so that

$$
d t=\hat{x}^{\prime}(c) d c=-\frac{d c}{1-F(\hat{x}(c))} .
$$

Noting that

$$
\hat{x}\left(c_{0}\left(p^{*}\right)\right)=\hat{x}\left(\min \left\{\bar{c}, \tilde{c}\left(p^{*}\right)\right\}\right)=\max \left\{\hat{x}(\bar{c}), p^{*}\right\}
$$

and

$$
\hat{x}\left(\max \left\{\underline{c}, \hat{c}\left(p_{i}\right)\right\}\right)=\min \left\{\hat{x}\left(\tilde{c}\left(p_{i}\right)\right), \hat{x}(\underline{c})\right\}=\min \left\{\bar{\varepsilon}-p_{i}+p^{*}, \hat{x}(\underline{c})\right\}
$$

we have

$$
D\left(p_{i}, p^{*}\right)=\left\{\begin{array}{cc}
\int_{\max \left\{\hat{x}(\bar{c}), p^{*}\right\}}^{\hat{x}(\underline{c})}\left(1-F\left(t+p_{i}-p^{*}\right)\right) g\left(\hat{x}^{-1}(t)\right) d t & \text { if } p_{i}<p^{*}, \\
\int_{\max \left\{\hat{x}(\bar{c}), p^{*}\right\}}^{\left.\min \left\{\bar{\varepsilon}-p_{i}+p^{*}, \hat{x}\right)\right\}}\left(1-F\left(t+p_{i}-p^{*}\right)\right) g\left(\hat{x}^{-1}(t)\right) d t & \text { if } p_{i} \geq p^{*} .
\end{array}\right.
$$


We now show that the integrand function

$$
\ell\left(p_{i}, t\right) \equiv\left(1-F\left(t+p_{i}-p^{*}\right)\right) g\left(\hat{x}^{-1}(t)\right)
$$

is jointly log-concave in $p_{i}$ and $t$ under the assumption that $g\left(\hat{x}^{-1}(t)\right)$ is log-concave in $t$. Because the product of log-concave functions is log-concave, $\ell\left(p_{i}, t\right)$ is jointly log-concave in $p_{i}$ and $t$ if and only if $1-F\left(t+p_{i}-p^{*}\right)$ is. Let $m\left(p_{i}, c\right) \equiv \ln \left[1-F\left(t+p_{i}-p^{*}\right)\right]$. Taking derivatives we have:

$$
\frac{\partial m}{\partial t}=\frac{\partial m}{\partial p_{i}}=-\frac{f\left(t+p_{i}-p^{*}\right)}{1-F\left(t+p_{i}-p^{*}\right)} .
$$

To construct the Hessian matrix, we now compute the necessary second order derivatives:

$$
\frac{\partial^{2} m}{\partial t^{2}}=\frac{\partial^{2} m}{\partial p_{i}^{2}}=\frac{\partial^{2} m}{\partial p_{i} \partial t}=-\frac{f^{\prime}\left(t+p_{i}-p^{*}\right)\left[1-F\left(t+p_{i}-p^{*}\right)\right]+f\left(t+p_{i}-p^{*}\right)^{2}}{\left[1-F\left(t+p_{i}-p^{*}\right)\right]^{2}} \leq 0,
$$

where the sign follows from the log-concavity of $f$. From these second derivatives it can readily be concluded that the Hessian matrix is negative semidefinite; this implies that $m\left(p_{i}, c\right)$ is concave in $p_{i}$ and $t$. By implication, $\ell\left(p_{i}, t\right)$ is log-concave in $p_{i}$ and $t$ on the convex set

$\left[r, p^{*}\right] \times\left[\max \left\{\hat{x}(\bar{c}), p^{*}\right\}, \hat{x}(\underline{c})\right] \cup\left\{\left(p_{i}, t\right): p_{i} \in\left[p^{*}, p^{m}\right], t \in\left[\max \left\{\hat{x}(\bar{c}), p^{*}\right\}, \min \left\{\bar{\varepsilon}-p_{i}+p^{*}, \hat{x}(\underline{c})\right\}\right]\right\}$ so $\ell\left(p_{i}, t\right)$ is $\log$-concave on $\left[r, p^{m}\right] \times\left[p^{*}, \bar{\varepsilon}\right]$ because from Prékopa (1971) we know that if a function is log-concave on a convex set and equal to zero elsewhere then the function is log-concave in the entire space.

We now invoke the following result by Prékopa (1973), which shows that integration preserves log-concavity.

Lemma 2. [Prékopa, 1973] Let $f(x, y)$ be a function of $n+m$ variables where $x$ is an $n$ component and $y$ is an $m$-component vector. Suppose that $f$ is log-concave in $R^{n+m}$ and let $A$ be a convex subset of $R^{m}$. Then the function of the variable $x$ :

$$
\int_{A} f(x, y) d y
$$

is log-concave in the entire space $R^{n}$.

Because $\ell\left(p_{i}, t\right)$ is log-concave in $p_{i}$ and $t$ and we are integrating for all $t$ in the convex set $\left[p^{*}, \bar{\varepsilon}\right]$, the theorem implies that the demand function (16) is log-concave in $p_{i}$. We then conclude that an equilibrium exists and is unique.

Proof of Proposition 1. Consider the function

$$
h(c)=-\frac{f(\hat{x}(c))}{1-F(\hat{x}(c))} .
$$


We know that

$$
h^{\prime}(c)=\frac{f^{\prime}(\hat{x}(c))[1-F(\hat{x}(c))]+f^{2}(\hat{x}(c))}{[1-F(\hat{x}(c))]^{3}} \geq 0
$$

by the log-concavity of $1-F$. Given this, result (A) follows directly from Theorem 1 in Hadar and Russell (1971).

Taking the derivative again we have:

$$
\begin{aligned}
h^{\prime \prime}(c) & =\frac{\left\{f^{\prime \prime}(\hat{x}(c))[1-F(\hat{x}(c))]+f(\hat{x}(c)) f^{\prime}(\hat{x}(c))\right\} \hat{x}^{\prime}(c)[1-F(\hat{x}(c))]^{3}}{[1-F(\hat{x}(c))]^{6}} \\
& +\frac{\left.\left\{f^{\prime}(\hat{x}(c))[1-F(\hat{x}(c))]+f^{2}(\hat{x}(c))\right)\right\} 3[1-F(\hat{x}(c))]^{2} f(\hat{x}(c)) \hat{x}^{\prime}(c)}{[1-F(\hat{x}(c))]^{6}} \\
& =-\frac{f^{\prime \prime}(\hat{x}(c))[1-F(\hat{x}(c))]^{2}+4 f(\hat{x}(c)) f^{\prime}(\hat{x}(c))[1-F(\hat{x}(c))]+3 f^{3}(\hat{x}(c))}{[1-F(\hat{x}(c))]^{5}} .
\end{aligned}
$$

Under the assumption $f^{\prime \prime}[1-F]^{2}+4 f f^{\prime}[1-F]+3 f^{3} \geq 0$ we know that $h(c)$ is an increasing and concave function. Given this, result (B) follows directly from Theorem 2 in Hadar and Russell (1971).

Proof of Proposition 3. We first deal with the case when $g(c ; \beta)$ has the ILRP. Denote

$$
\tilde{G}(c ; \beta)=G\left(c ; \beta \mid c \leq \tilde{c}\left(p^{*}\right)\right), \quad \tilde{g}(c ; \beta)=g\left(c ; \beta \mid c \leq \tilde{c}\left(p^{*}\right)\right)
$$

so

$$
\tilde{G}(c ; \beta)=\frac{G(c ; \beta)}{G\left(\tilde{c}\left(p^{*}\right) ; \beta\right)}, \quad \tilde{g}(c ; \beta)=\frac{g(c ; \beta)}{G\left(\tilde{c}\left(p^{*}\right) ; \beta\right)}
$$

where

$$
G\left(\tilde{c}\left(p^{*}\right) ; \beta\right)=\int_{\underline{c}(\beta)}^{\tilde{c}\left(p^{*}\right)} g(c ; \beta) d c
$$

We now prove that

$$
\int_{\underline{c}(\beta)}^{\tilde{c}\left(p^{*}\right)} h(c) \tilde{g}(c ; \beta) d c \geq \int_{\underline{c}(\beta)}^{\tilde{c}\left(p^{*}\right)} h(c) \tilde{g}(c ; \gamma) d c
$$

for any decreasing function $h$ and $\gamma \geq \beta$. This is equivalent to showing that for any decreasing differentiable function $h$ we have that

$$
\frac{\int_{\underline{c}(\beta)}^{\tilde{c}\left(p^{*}\right)} h(c) g(c ; \beta) d c}{\int_{\underline{c}(\beta)}^{\tilde{c}\left(p^{*}\right)} g(c ; \beta) d c} \geq \frac{\int_{\underline{c}(\beta)}^{\tilde{c}\left(p^{*}\right)} h(c) g(c ; \gamma) d c}{\int_{\underline{c}(\beta)}^{\tilde{c}\left(p^{*}\right)} g(c ; \gamma) d c} .
$$

To prove equation (18), we make use of Lemma 1. Let

$$
\begin{aligned}
& f_{1}(c)=g(c, \beta), \quad f_{2}(c)=h(c) g(c, \gamma), \\
& f_{3}(c)=g(c, \gamma), \quad f_{4}(c)=h(c) g(c, \beta) .
\end{aligned}
$$


We show that for all $c, d \in\left[0, \tilde{c}\left(p^{*}\right)\right]$

$$
f_{1}(c) f_{2}(d) \leq f_{3}(\max \{c, d\}) f_{4}(\min \{c, d\})
$$

i.e.,

$$
g(c, \beta) h(d) g(d, \gamma) \leq g(\max \{c, d\}, \gamma) h(\min \{c, d\})) g(\min \{c, d\}, \beta) .
$$

Take first $c<d$. Because $\max \{c, d\}=d$ and $\min \{c, d\}=c,(19)$ simplifies to

$$
g(c, \beta) h(d) g(d, \gamma) \leq g(d, \gamma) h(c) g(c, \beta)
$$

which is true because $h$ is decreasing in $c$ and $c<d$.

Now take $c \geq d$; we have $\max \{c, d\}=c, \min \{c, d\}=d$. In this case, (19) holds whenever

$$
g(c, \beta) h(d) g(d, \gamma) \leq g(c, \gamma) h(d) g(d, \beta)
$$

or reorganizing

$$
h(d)[g(c, \beta) g(d, \gamma)-g(c, \gamma) g(d, \beta)] \leq 0,
$$

which clearly holds for all $c \geq d$ if and only if $g(c, \beta)$ has the ILRP. ${ }^{13}$ This shows that (17) holds.

Rewrite now (17) as

$$
\int_{\underline{c}(\beta)}^{\tilde{c}\left(p^{*}\right)} h(c)[\tilde{g}(c ; \gamma)-\tilde{g}(c ; \beta)] d c=\int_{\underline{c}(\beta)}^{\tilde{c}\left(p^{*}\right)} h(c) d[\tilde{G}(c ; \gamma)-\tilde{G}(c ; \beta)] \leq 0 .
$$

By integration by parts we have

$$
\begin{array}{r}
\left.h(c)[\tilde{G}(c ; \gamma)-\tilde{G}(c ; \beta)]\right|_{0} ^{\tilde{c}\left(p^{*}\right)}-\int_{\underline{c}(\beta)}^{\tilde{c}\left(p^{*}\right)} h^{\prime}(c)[\tilde{G}(c ; \gamma)-\tilde{G}(c ; \beta)] d c \\
=-\int_{\underline{c}(\beta)}^{\tilde{c}\left(p^{*}\right)} h^{\prime}(c)[\tilde{G}(c ; \gamma)-\tilde{G}(c ; \beta)] d c \leq 0 .
\end{array}
$$

Since this holds for arbitrary $h$ for which $h^{\prime}(c) \leq 0$, it implies that $\tilde{G}(c ; \gamma) \leq \tilde{G}(c ; \beta)$ for any $c$.

For the case of densities that satisfy the DLRP, we prove that

$$
\int_{\underline{c}(\beta)}^{\tilde{c}\left(p^{*}\right)} h(c) \tilde{g}(c ; \beta) d c \leq \int_{\underline{c}(\beta)}^{\tilde{c}\left(p^{*}\right)} h(c) \tilde{g}(c ; \gamma) d c
$$

for any decreasing function $h$, which is equivalent to showing that for any decreasing differentiable function $h$ we have that

$$
\frac{\int_{\underline{c}(\beta)}^{\tilde{c}\left(p^{*}\right)} h(c) g(c ; \beta) d c}{\int_{\underline{c}(\beta)}^{\tilde{c}\left(p^{*}\right)} g(c ; \beta) d c} \leq \frac{\int_{\underline{c}(\beta)}^{\tilde{c}\left(p^{*}\right)} h(c) g(c ; \gamma) d c}{\int_{\underline{c}(\beta)}^{\tilde{c}\left(p^{*}\right)} g(c ; \gamma) d c} .
$$

\footnotetext{
${ }^{13}$ If $g(c, \beta)=0$ then the inequality clearly holds for any $d$. If $g(d, \beta)=0$ then $d=0$. Then the fact $g(d, \beta)=0$ means that the derivative of $G(c, \beta)$ is 0 at 0 . Since from FOSD (implied by the ILRP) $G(c, \gamma) \leq G(c, \beta)$, the derivative of $G(c, \gamma)$ must also be 0 at 0 . Therefore, $g(d, \gamma)=0$, so the inequality holds.
} 
We can again invoke Lemma 1 and show that this is true for densities with the DLRP. The implication is that $\tilde{G}(c ; \gamma) \geq \tilde{G}(c ; \beta)$ for any $c$.

Example 1. We first need to prove that an equilibrium exists and is unique for the search cost density

$$
g(c)=\frac{\beta(2+\delta)-2 c}{\beta^{2}(1+\delta)}, \text { with } \delta \geq 0
$$

Because match values are uniformly distributed on $[0,1]$, for existence and uniqueness of symmetric equilibrium we only need to check that the function $g\left(\hat{x}^{-1}(t)\right)$ is log-concave in $t$.

Solving the consumers equilibrium condition (1) gives $\hat{x}(c)=1-\sqrt{2 c}$; therefore $\hat{x}^{-1}(t)=$ $(1-t)^{2} / 2$. Plugging this into the density function gives

$$
g\left(\hat{x}^{-1}(t)\right)=\frac{(1-t)^{2}-\beta(2+\delta)}{\beta^{2}(1+\delta)} .
$$

Inspection of this function immediately reveals that it is strictly concave in $t$; therefore it is log-concave in $t$. We conclude that an equilibrium exists an is unique.

We now show that $g$ satisfies the ILRP with respect to parameters $\beta$ and $\delta$. From Milgrom (1981), $g$ has the ILRP with respect to parameter $\beta$ if and only if the ratio $g_{\beta}^{\prime} / g$ is increasing in $c$, where $g_{\beta}^{\prime}$ denotes the derivative of $g$ with respect to $\beta$. We have

$$
\frac{g_{\beta}^{\prime}}{g}=\frac{4 c-\beta(2+\delta)}{\beta(\beta(2+\delta)-2 c)},
$$

and

$$
\frac{d\left(g_{\beta}^{\prime} / g\right)}{d c}=\frac{2(2+\delta)}{(\beta(2+\delta)-2 c)^{2}}>0 .
$$

For the case of parameter $\delta$ we have

$$
\frac{g_{\delta}^{\prime}}{g}=\frac{\beta}{\beta(2+\delta)-2 c}-\frac{1}{1+\delta},
$$

and

$$
\frac{d\left(g_{\delta}^{\prime} / g\right)}{d c}=\frac{2 \beta}{(\beta(2+\delta)-2 c)^{2}}>0 .
$$

Finally we observe that an increase in either parameter $\beta$ or $\delta$ signifies a FOSD shift of the search cost CDF. For this we compute

$$
\frac{d G}{d \beta}=-\frac{c(\beta(2+\delta)-2 c)}{\beta^{3}(1+\delta)}<0
$$

and

$$
\frac{d G}{d \delta}=-\frac{c(\beta-c)}{\beta^{2}(\delta+1)^{2}}<0
$$


The details of the example are now complete.

Example 2. Consider now the case when search costs are distributed on $[0, \beta]$ according to the density

$$
g(c ; \beta)=\frac{1+\delta}{\beta(2+\delta)}\left[1+\left(\frac{c}{\beta}\right)^{\delta}\right], \text { with } 0<\delta \leq 1 / 2,
$$

and again assume match values are distributed uniformly on $[0,1]$.

For this density function we have

$$
g\left(\hat{x}^{-1}(t)\right)=\frac{(1+\delta)\left(2^{\delta}+\left(\frac{(1-t)^{2}}{\beta}\right)^{\delta}\right)}{2^{\delta} \beta(2+\delta)} .
$$

Taking the second derivative with respect to $t$ gives

$$
\frac{d^{2} g\left(\hat{x}^{-1}(t)\right)}{d t^{2}}=\frac{2^{1-\delta} \delta\left(1-\delta-2 \delta^{2}\right)(1-t)^{2 \delta-2}}{\beta^{1+\delta}(2+\delta)}
$$

which is clearly negative for $0<\delta \leq 1 / 2$. Therefore $g\left(\hat{x}^{-1}(t)\right)$ is concave in $t$ and by implication it is log-concave in $t$. We conclude that an equilibrium exists and is unique.

We now observe that $g$ has the DLRP with respect to parameter $\beta$. To see this, we note that

$$
\frac{g_{\beta}^{\prime}}{g}=\frac{-\frac{1+\delta+(1+\delta)^{2}\left(\frac{c}{\beta}\right)^{\delta}}{\beta^{2}(2+\delta)}}{\frac{1+\delta}{\beta(2+\delta)}\left[1+\left(\frac{c}{\beta}\right)^{\delta}\right]}=-\frac{1+(1+\delta)\left(\frac{c}{\beta}\right)^{\delta}}{\beta\left[1+\left(\frac{c}{\beta}\right)^{\delta}\right]}
$$

Taking the derivative with respect to $c$ gives

$$
\frac{\partial\left(g_{\beta}^{\prime} / g\right)}{\partial c}=-\frac{\delta^{2}\left(\frac{c}{\beta}\right)^{\delta-1}}{\beta^{2}\left[1+\left(\frac{c}{\beta}\right)^{\delta}\right]^{2}}<0 .
$$

Finally, we note an increase in $\beta$ shifts the search cost distribution to the right, so higher $\beta$ implies a FOSD shift of the search cost distribution. This is seen directly form the following derivative:

$$
\frac{d G}{d \beta}=-\frac{c(1+\delta)\left(1+\left(\frac{c}{\beta}\right)^{\delta}\right)}{\beta^{2}(2+\delta)}<0 .
$$

The details of the example are not complete.

\section{Duopoly.}


In this Appendix we study the duopoly case. Except that there are only two firms in the market, the rest of the model details are exactly the same as before. ${ }^{14}$

We now present the derivations to compute the SNE price $p^{*}$. For this we derive the (expected) payoff to a firm $i$ that deviates by charging a price $p_{i}<p^{*}$. In order to compute firm $i$ 's demand, consider a consumer with search cost $c$ who visits firm $i$ in her first search. This happens with probability $1 / 2$. Let $\varepsilon_{i}-p_{i}$ denote the utility the consumer derives from the product of firm $i$. Notice that search behavior is exactly the same as in the previous section. The consumer expects the other firm to charge the equilibrium price $p^{*}$. The expected gains from searching one more time are equal to $\int_{\varepsilon_{i}-p_{i}+p^{*}}^{1}\left[\varepsilon_{j}-\left(\varepsilon_{i}-p_{i}+p^{*}\right)\right] f\left(\varepsilon_{j}\right) d \varepsilon_{j}$. It follows that the probability that the buyer visits firm $i$ first and stops searching at firm $i$ is equal to

$$
\frac{1}{2} \operatorname{Pr}\left[\varepsilon_{i}-p_{i}>\max \left\{\hat{x}(c)-p^{*}, 0\right\}\right]=\frac{1}{2}\left[1-F\left(\hat{x}(c)+p_{i}-p^{*}\right)\right] .
$$

where $\hat{x}(c)$ continues to be the solution to equation (1). Consumer $c$ may find the product of firm $i$ not good enough at first and may therefore continue searching. However, upon visiting the rival firm $j$, it may happen that consumer $c$ returns to firm $i$ because such a firm offers her the best deal after all. This occurs with probability

$$
\frac{1}{2} \operatorname{Pr}\left[\max \left\{\varepsilon_{j}-p^{*}, 0\right\}<\varepsilon_{i}-p_{i}<\hat{x}(c)-p^{*}\right]=\frac{1}{2} \int_{p_{i}}^{\hat{x}(c)+p_{i}-p^{*}} F\left(\varepsilon-p_{i}+p^{*}\right) f(\varepsilon) d \varepsilon .
$$

With probability $1 / 2$ consumer $c$ first visits the other firm, firm $j$. In that case, she will walk away from product $j$ when searching again is more promising than buying $j$ right away. Upon visiting firm $i$, she will buy product $i$ when she finds product $i$ better than $j$. This occurs with probability $(1 / 2) \operatorname{Pr}\left[\max \left\{\varepsilon_{j}-p^{*}, 0\right\}<\min \left\{\hat{x}(c)-p^{*}, \varepsilon_{i}-p_{i}\right\}\right]$, which is equal to

$$
\frac{1}{2}\left[F(\hat{x}(c))\left[1-F\left(\hat{x}(c)+p_{i}-p^{*}\right)\right]+\int_{p_{i}}^{\hat{x}(c)+p_{i}-p^{*}} F\left(\varepsilon-p_{i}+p^{*}\right) f(\varepsilon) d \varepsilon\right] .
$$

To obtain the payoff of firm $i$ we need to integrate over the consumers who decide to participate in the market. It can be shown that with two firms the surplus of a consumer with search cost $c$ is given by the expression

$$
C S(c)=\frac{1-F(\hat{x}(c))^{2}}{1-F(\hat{x}(c))}\left[\int_{\hat{x}(c)}^{\bar{\varepsilon}}\left(\varepsilon-p^{*}\right) f(\varepsilon) d \varepsilon-c\right]+2 \int_{p^{*}}^{\hat{x}(c)}\left(\varepsilon-p^{*}\right) F(\varepsilon) f(\varepsilon) d \varepsilon .
$$

Setting this surplus equal to zero, we obtain the critical search cost value $\tilde{c}\left(p^{*}\right)$ above which consumers will refrain from participating in the market. Inspection of equation (23) reveals that

\footnotetext{
${ }^{14}$ Extending this analysis to the case of $N$ firms is straightforward.
} 
the last consumer who chooses to search has a search cost $c$ such that $\hat{x}(c)=p^{*}$. Using again the notation $c_{0}\left(p^{*}\right) \equiv \min \left\{\bar{c}, \tilde{c}\left(p^{*}\right)\right\}$, the expected payoff to firm $i$ is:

$$
\pi_{i}\left(p_{i} ; p^{*}\right)=\frac{p_{i}-r}{2} \int_{0}^{c_{0}\left(p^{*}\right)}\left[(1+F(\hat{x}(c)))\left(1-F\left(\hat{x}(c)+p_{i}-p^{*}\right)\right)+2 \int_{p_{i}}^{\hat{x}(c)+p_{i}-p^{*}} F\left(\varepsilon-p_{i}+p^{*}\right) f(\varepsilon) d \varepsilon\right] g(c) d c .
$$

To shorten the expressions we will from now on write $\hat{x}$ instead of $\hat{x}(c)$ but the reader should keep in mind the dependency of $\hat{x}$ on $c$. Taking the FOC gives

$$
\begin{aligned}
0 & =\int_{0}^{c_{0}\left(p^{*}\right)}\left[(1+F(\hat{x}))\left[1-F\left(\hat{x}+p_{i}-p^{*}\right)\right]+2 \int_{p_{i}}^{\hat{x}+p_{i}-p^{*}} F\left(\varepsilon-p_{i}+p^{*}\right) f(\varepsilon) d \varepsilon\right] g(c) d c \\
& -\left(p_{i}-r\right) \int_{0}^{c_{0}\left(p^{*}\right)}(1+F(\hat{x})) f\left(\hat{x}+p_{i}-p^{*}\right) g(c) d c \\
& -2\left(p_{i}-r\right) \int_{0}^{c_{0}\left(p^{*}\right)}\left[\int_{p_{i}}^{\hat{x}+p_{i}-p^{*}} f\left(\varepsilon-p_{i}+p^{*}\right) f(\varepsilon) d \varepsilon+F(\hat{x}) f\left(\hat{x}+p_{i}-p^{*}\right)-F\left(p^{*}\right) f\left(p_{i}\right)\right] g(c) d c .
\end{aligned}
$$

Applying symmetry $p_{i}=p^{*}$ gives

$$
\begin{aligned}
0 & =\int_{0}^{c_{0}\left(p^{*}\right)}\left[(1+F(\hat{x}))(1-F(\hat{x}))+2 \int_{p^{*}}^{\hat{x}} F(\varepsilon) f(\varepsilon) d \varepsilon\right] g(c) d c \\
& -2\left(p^{*}-r\right) \int_{0}^{c_{0}\left(p^{*}\right)}\left[(1+F(\hat{x})) f(\hat{x})+\int_{p^{*}}^{\hat{x}} f(\varepsilon)^{2} d \varepsilon-F(\hat{x}) f(\hat{x})+F\left(p^{*}\right) f\left(p^{*}\right)\right] g(c) d c .
\end{aligned}
$$

In order to check how the equilibrium price changes when search costs go up, we proceed by solving the FOC in equation (26) numerically. We again use the uniform distribution for the match values. For search costs we use the Kumaraswamy distribution:

Definition: The Kumaraswamy distribution has $C D F G(\cdot)$ and $P D F g(\cdot)$ given by

$$
\begin{aligned}
& G(c)=1-\left[1-\left(\frac{c}{\beta}\right)^{a}\right]^{b}, c \in[0, \beta], a, b>0 ; \\
& g(c)=\frac{a b}{\beta}\left(\frac{c}{\beta}\right)^{a-1}\left[1-\left(\frac{c}{\beta}\right)^{a}\right]^{b-1} .
\end{aligned}
$$

The Kumaraswamy (1980) distribution is often used as a substitute for the beta-distribution (see, e.g., Ding and Wolfstetter, 2011). This distribution turns out to be quite useful in our setting because its likelihood ratio is increasing (for $b>1$ ), decreasing (for $0<b<1$ ), or constant (for $b=1$ ) with respect to the shifter parameter $\beta .{ }^{15}$ Note that the $\beta$ parameter multiplies the search cost $c$ and scales the support of the distribution. An increase in $\beta$ therefore shifts the search cost distribution rightward, which signifies that search costs are higher for all consumers.

\footnotetext{
${ }^{15} \mathrm{~A}$ proof can be obtained from the authors upon request. Observe that the uniform density case is obtained by setting $a=b=1$ in the Kumaraswamy distribution above.
} 
The focus is on the case in which the upper bound of the search cost distribution $\beta$ is sufficiently high (Theorem 1B).

For the case of the uniform distribution we have that

$$
C S(c)=\hat{x}(c)-p^{*}-\frac{\hat{x}(c)^{3}-p^{* 3}}{3},
$$

whereas the critical search cost value above which consumers refrain from searching the market is

$$
\tilde{c}\left(p^{*}\right)=\frac{\left(1-p^{*}\right)^{2}}{2}
$$

In Table 1, we set $r=0$ and let search costs be distributed according to the Kumaraswamy distribution with parameter $a=1$ and various levels of the parameters $b$ and $\beta$. The table shows once again that prices decrease when search costs increase when $b=1 / 2$, in which case the search cost density has the MDLR property. For the $b=1$ case (uniform distribution), once more prices are independent of the search cost upper bound. Finally, when $b=3 / 2$ and the search cost density has the MILR property, we get the standard result that prices increase with higher search costs.

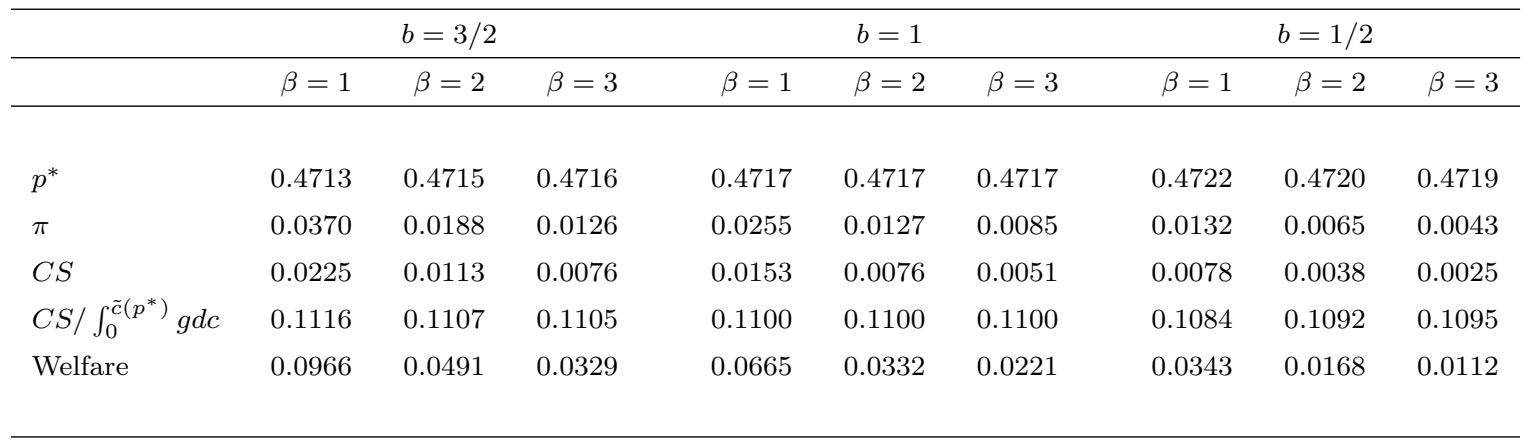

Table 1: Duopoly model (uniform-Kumaraswamy with $a=1$ ) 


\section{References}

Ahlswede, Rudolf and David E. Daykin: "An Inequality for Weights of Two Families of Sets, their Unions and Intersections," Zeitschrift für Wahrscheinlichkeitstheorie und Verwandte Gebiete 43, 183-185, 1978.

Anderson, Simon P. and Régis Renault: "Pricing, Product Diversity, and Search Costs: A Bertrand-Chamberlin-Diamond Model," RAND Journal of Economics 30, 719-735, 1999.

Anderson, Simon P. and Régis Renault: "Advertising Content," American Economic Review 96, 93-113, 2006.

Armstrong, Mark, John Vickers, and Jidong Zhou: "Prominence and Consumer Search," RAND Journal of Economics 40, 209-33, 2009.

Armstrong, Mark, and Jidong Zhou: "Paying for Prominence," Economic Journal 121, F368F395, 2011.

Athey, Susan: "Monotone Comparative Statics under Uncertainty." Quarterly Journal of Economics 117-1, 187-223, 2002.

Bar-Isaac, Heski, Guillermo Caruana, and Vicente Cuñat: "Search, Design and Market Structure," American Economic Review 102, 1140-1160, 2012.

Bénabou, Roland, "Search Market Equilibrium, Bilateral Heterogeneity, and Repeat Purchases," Journal of Economic Theory 60, 140-158, 1993.

Brynjolfsson, Erik, Yu (Jeffrey) Hu, and Duncan Simester: "Goodbye Pareto Principle, Hello Long Tail: The Effect of Search Costs on the Concentration of Product Sales," Management Science 57, 1373-1386, 2011.

Brynjolfsson, Erik and Michael D. Smith: "Frictionless Commerce? A Comparison of Internet and Conventional Retailers," Management Science 46, 563-85, 2000.

Burdett, Kenneth and Kenneth L. Judd: "Equilibrium Price Dispersion," Econometrica 51, 955-969, 1983.

Caplin, Andrew and Berry Nalebuff: "Aggregation and Imperfect Competition: On the Existence of Equilibrium," Econometrica 59, 25-59, 1991. 
Chen, Yongmin and Tianle Zhang: "Equilibrium Price Dispersion with Heterogeneous Searchers," International Journal of Industrial Organization 29, 645-654, 2011.

Clay, Karen, Ramayya Krishnan and Eric Wolff: "Prices and Price Dispersion on the Web: Evidence from the Online Book Industry," Journal of Industrial Economics 49, 521-540, 2001.

Clemons, Eric K., Il-Horn Hann, and Lorin M. Hitt: "Price Dispersion and Differentiation in Online Travel: An Empirical Investigation," Management Science 48, 534-549, 2002.

Diamond, Peter A.: "A Model of Price Adjustment," Journal of Economic Theory 3, 156-168, 1971.

Ding, W. and E. Wolfstetter: "Prizes and Lemons: Procurement of Innovation under Imperfect Commitment," RAND Journal of Economics 42, 664-680, 2011.

Ellison, Glenn and Alexander Wolitzky: "A Search Cost Model of Obfuscation," The RAND Journal of Economics 43, 417-441, 2012.

Goolsbee, Austan: "Competition in the Computer Industry: Online Versus Retail," Journal of Industrial Economics 49, 487-499, 2001.

Haan, Marco A. and José Luis Moraga-González: "Advertising for Attention in a Consumer Search Model," The Economic Journal 121, 552-579, 2011a.

Haan, Marco A., and José Luis Moraga-González: "Consumer Search with Observable and Hidden Characteristics," Mimeo, 2011b.

Hadar, Josef and William R. Russell: "Stochastic Dominance and Diversification," Journal of Economic Theory 3, 288-305, 1971.

Hortaçsu, Ali and Chad Syverson: "Product Differentiation, Search Costs, and Competition in the Mutual Fund Industry: A Case Study of S\&P 500 Index Funds," Quarterly Journal of Economics 119, 403-456, 2004.

Janssen, Maarten C.W., José Luis Moraga-González, and Matthijs R. Wildenbeest: "Truly Costly Sequential Search and Oligopolistic Pricing," International Journal of Industrial Organization 23, 451-466, 2005.

Janssen, Maarten C.W. and Sandro Shelegia: "Consumer Search and Double Marginalization," American Economic Review 105 (6), 2015, 1-29. 
Kohn, Meir G. and Steven Shavell: "The Theory of Search," Journal of Economic Theory 9, 93-123, 1974.

Kumaraswamy, P.: "A Generalized Probability Density Function for Double-Bounded RandomProcesses," Journal of Hydrology 46, 79-88, 1980.

Lee, Ho Geun: "Do Electronic Marketplaces Lower the Price of the Goods?," Communications of the ACM 41, 73-80, 1998.

Moraga-González, José Luis and Vaiva Petrikaitè: "Search Costs, Demand-Side Economies and the Incentives to Merge under Bertrand Competition," RAND Journal of Economics 44, 391-424, 2013.

Milgrom, Paul R.:"Good News and Bad News: Representation Theorems and Applications," The Bell Journal of Economics 12, 380-391, 1981.

Prékopa, András: "Logarithmic Concave Measures with Application to Stochastic Programming," Acta Scientiarum Mathematicarum 32, 301-316, 1971.

Prékopa, András: "On Logarithmic Concave Measures and Functions," Acta Scientiarum Mathematicarum 34, 335-343, 1973.

Rauh, Michael T.: "Wage and Price Controls in the Equilibrium Sequential Search Model," European Economic Review 48, 1287-1300, 2004.

Rauh, Michael T.: "Strategic Complementarities and Search Market Equilibrium," Games and Economic Behavior 66, 959-978, 2009.

Rob, Rafael: "Equilibrium Price Distributions," Review of Economic Studies 52, 452-504, 1985.

Scott Morton, Fiona, Florian Zettelmeyer, and Jorge Silva-Risso: "Internet Car Retailing," Journal of Industrial Economics 49, 509-519, 2001.

Stahl, Dale O.: "Oligopolistic Pricing with Sequential Consumer Search," American Economic Review 79, 700-712, 1989.

Stahl, Dale O.: "Oligopolistic Pricing with Heterogeneous Consumer Search," International Journal of Industrial Organization 14, 243-268, 1996.

Stiglitz, Joseph E.: "Equilibrium in Product Markets with Imperfect Information," American Economic Review 69, 339-345, 1979. 
Tappata, Mariano: "Rockets and Feathers: Understanding Asymmetric Pricing," The RAND Journal of Economics 40, 673-687, 2009.

Wilson, Chris M.: "Ordered Search and Equilibrium Obfuscation," International Journal of Industrial Organization 28, 496-506, 2010.

Wolinsky, Asher: "True Monopolistic Competition as a Result of Imperfect Competition," Quarterly Journal of Economics 101, 493-511, 1986.

Zhou, Jidong: "Multiproduct Search and the Joint Search Effect," American Economic Review, 104-9, 2918-2939, 2014. 